\title{
SiSeRHMap v1.0: a simulator for mapped seismic response using a hybrid model
}

\author{
Gerardo Grelle $^{1}$, Laura Bonito ${ }^{2}$, Alessandro Lampasi ${ }^{3}$, Paola Revellino ${ }^{2}$, Luigi Guerriero ${ }^{2}$, Giuseppe Sappa ${ }^{1}$, and \\ Francesco Maria Guadagno ${ }^{2}$ \\ ${ }^{1}$ Department of Civil and Environmental Engineering, University of Rome "La Sapienza” via Eudossiana 18, \\ 00184, Rome, Italy \\ ${ }^{2}$ Department of Sciences and Technologies, University of Sannio, via Dei Mulini 59/A, 82100, Benevento, Italy \\ ${ }^{3}$ ENEA Frascati Research Center, Via Enrico Fermi 45, 00044 Frascati, Rome, Italy
}

Correspondence to: Gerardo Grelle (gerardo.grelle@unisannio.it, gerardo.grelle@uniroma1.it)

Received: 19 March 2015 - Published in Geosci. Model Dev. Discuss.: 17 June 2015

Revised: 25 March 2016 - Accepted: 8 April 2016 - Published: 26 April 2016

\begin{abstract}
The SiSeRHMap (simulator for mapped seismic response using a hybrid model) is a computerized methodology capable of elaborating prediction maps of seismic response in terms of acceleration spectra. It was realized on the basis of a hybrid model which combines different approaches and models in a new and non-conventional way. These approaches and models are organized in a code architecture composed of five interdependent modules. A GIS (geographic information system) cubic model (GCM), which is a layered computational structure based on the concept of lithodynamic units and zones, aims at reproducing a parameterized layered subsoil model. A meta-modelling process confers a hybrid nature to the methodology. In this process, the one-dimensional (1-D) linear equivalent analysis produces acceleration response spectra for a specified number of site profiles using one or more input motions. The shear wave velocity-thickness profiles, defined as trainers, are randomly selected in each zone. Subsequently, a numerical adaptive simulation model (Emul-spectra) is optimized on the above trainer acceleration response spectra by means of a dedicated evolutionary algorithm (EA) and the Levenberg-Marquardt algorithm (LMA) as the final optimizer. In the final step, the GCM maps executor module produces a serial map set of a stratigraphic seismic response at different periods, grid solving the calibrated Emul-spectra model. In addition, the spectra topographic amplification is also computed by means of a 3 -D validated numerical prediction model. This model is built to match the results of the numerical simulations related to isolate reliefs using GIS morphometric data. In this
\end{abstract}

way, different sets of seismic response maps are developed on which maps of design acceleration response spectra are also defined by means of an enveloping technique.

\section{Introduction}

In the scientific community, it is well known that lithologic stratigraphy as well as topographic features are capable of considerably amplifying the local destructive action of an earthquake (Del Prete et al., 1998; Athanasopoulos et al., 1999). Thus, in prone areas, seismic microzonation studies assume an important role in urban planning and seismic risk management (Lachet et al., 1996; Bianchi Fasani et al., 2008; Compagnoni et al., 2011; Milana et al., 2011; Grasso and Maugeri, 2012; Moscatelli et al., 2014). As a consequence, methods for high levels of seismic microzonation (mapped seismic response studies) aim at providing quantitative data for use in building design (Borcherdt, 1994; Todd and Harris, 1995; Bostenaru Dan, 2005; Kokošin and Gosar, 2013). Many building codes, such as Euro Code 8 and FEMA 356 (2000), require seismic design actions defined by simplified elastic acceleration spectra deriving from local base seismic hazard (as reference natural or virtual stiff rock site which are defined in term of horizontal acceleration probability of exceedance in specified time interval) and site amplification effects.

In addition to a need to have a sufficient amount of information suitable for seismic microzonation, computerized 
data management and spatial distribution in terms of input and output/outcomes, are also required. Therefore, the geographic information systems (GIS) contribute the most to maximizing the available data, in the assessment or estimation of ground-motion amplification (Kolat et al., 2006; Ganapathy, 2011; Hashemi and Alesheikh, 2012; Turk et al., 2012; Hassanzadeh et al., 2013) and seismic-induced effects (Grelle et al., 2011; Grelle and Guadagno, 2013).

In this aforementioned context, SiSeRHMap (simulator for mapped seismic response using a hybrid model) provides synthetic multi-map data regarding a complex phenomenon, such as seismic site response, on the basis of a new hybrid methodology in which a meta-modelling process is the core feature. In recent years, the use of meta-models in many engineering and environmental science fields (Lampasi et al., 2006; Yazdi and Neyshabouri, 2014; Wang et al., 2014; Hong et al., 2014), together with GIS supported analysis (Reed et al., 2012; Fan et al., 2015; Soares et al., 2014), has produced good performances, providing fast versatility and rapid updating.

By nature hybrid systems based on meta-models include intrinsic uncertainty in their predictions. This is due to the use of non-physical adaptive models trained on simplified physical models. On the other hand, these systems permit an efficient analysis in terms of expected performance. Essentially, a meta-model permits a quick replication of the solutions in a limited context of randomness. In this way the proposed model is very suitable for a continual easy modular update that decreases the epistemic uncertainty, over time, in the assessment of the effects of natural complex phenomena, such as seismic response, on a real natural system. Therefore, SiSeRHMap is formulated on the concept of "performance", regarding (i) prediction, (ii) easy and low computational time, (iii) upgrading, and (iv) output accessibility (GIS-georeferenced data), with respect to the real effect. For these reasons, SiSeRHMap aims at giving a substantial contribution to common practices. Contextualized for a practical application in site seismic response studies, limits of usual practice may be currently summarized as (i) a partial contribution of the microzonation study with regards to providing appropriate quantitative parameters for seismic engineering practice; (ii) an inadequate use of some simplified amplified design spectra defined by means of some large ranges of $V_{\mathrm{S}}$ that refer to $30 \mathrm{~m}$ or to the deep bedrock; (iii) an unsuitable use of the point-data spatial interpolation for the mapped seismic response values.

Considering the aforementioned critical issues, in areas with a not very high geological complexity, the proposed methodology can present a high computational efficiency in comparison to expensive rigorous physically based models; this efficiency multiplies when a probability multi-input motion analysis is performed. Therefore, the map-sets of seismic response provided by SiSeRHMap are the result of an advantageous compromise between intrinsic and epistemic uncertainties and the accuracy and robustness required. This last aspect reflects the aptitude of the proposed methodology, which is suitable for analysis of urban areas or relatively vast areas. In general the level of accuracy of the SiSeRHMap response increases with the number and quality of the surveys; however, it is suitable to be used in areas with common and non-strategic facilities (e.g. nuclear plants); for strategic facilities, a detailed analysis may be required due to the fact that the use of a meta-model might not ensure the level of accuracy required.

\subsection{Code design and aims}

SiSeRHMap is a computer program methodology aimed at the mapped simulator for mapped seismic response using a hybrid model. The hybrid model consists of a complex computational system composed of a GIS frame model, analytical models (physically based) and meta-modelling procedures. SiSeRHMap is capable of developing map sets of seismic response taking into account the combined effects of plane-parallel stratigraphy and real topographic features. It is composed of five progressive interdepending Python compute modules, each of which necessitates external input data. The input data and data set are inserted or linked into a textual user interface (TUI) which writes the file "Instruction.txt" that the Python modules read in running.

The modules and their computational functions are as follows:

- mod.1: lithodynamic units parameterization;

- mod.2: GIS cubic model frame;

- mod.3: stratigraphic response;

- mod.4: training "spectra";

- mod.5: GCM maps executor.

\subsection{Background}

In mapped seismic response studies carried out using analytical methods for assessing or estimating stratigraphic seismic site responses, GIS provide the spatial distribution of parameters, which characterize the seismic motion (Jimenez et al., 2000; Sokolov and Chernov, 2001; Nath, 2004; Kienzle et al., 2006). Based on a multi-variate regression analysis of common recurrent regional data settings regarding simple sequences, procedures for calculating seismic soil response have also been introduced (Rodríguez-Marek et al., 2001; Papadimitriou et al., 2008).

Among the above-mentioned GIS-based models, Grelle et al. (2014) have recently introduced a hybrid model, based on the "GIS cubic model (GCM)" frame which is, in turn, based on the concept of lithodynamic units and zones. Here, a lithodynamic unit is defined as a lithological unit, which is characterized by a shear wave velocity-depth-dependent curve (as shown in Fig. 1) and subsequently by non-linear 
stress-strain behaviour. The zone is defined by a specific combination, in sequence, of lithodynamic units. The hybrid model computes the mapping of seismic response using an adaptive model, which is trained on one-dimensional (1-D) seismic response target cases calculated from some shear wave velocity-thickness sequences. These latter are uniformly randomly selected in coherence with general lithodynamic layered models assumed for the study area. In this way, the trained adaptive model, conceptually defined as a meta-model (replacement model), is used in the spatial predictive analysis, which aims at developing seismic response maps by means of its meta-model solving in the GCM.

Topographic amplification is a more relevant frequency dependent effect in zones characterized by hill and mountain features (Çelebi, 1987; Kawase and Aki, 1990; Assimaki et al., 2005; Del Gaudio and Wasowski, 2007; Hough et al., 2010; Massa et al., 2010; Pischiutta et al., 2010). 2-D and 3D simulation analytical approaches on different relief shapes, as well as different incident seismic wave motions, have been introduced (Sánchez-Sesma, 1983; Geli et al., 1988; Ashford et al., 1997; Durand et al., 1999; Maufroy et al., 2012, 2015). Geli et al. (1988) used numerical methods for assessing the topographic amplification factor, $A_{\mathrm{T}}$, of the vertical incident of horizontal shear waves (SH) on 2-D isolated reliefs constituted by uniform material and different layering structures. Their results highlighted that the frequency-dependent amplification factors change considerably along the topographic surface, showing a greater amplification at the ridge, reaching values over 2.00 in some cases. Ashford et al. (1997) quantified the theoretical effect of the horizontal and vertical seismic response at a ridge of monoclinal slopes, which is half-space extensive, by taking into consideration vertical incident SH waves. The analytical model assumes that the slopes are constituted by uniform viscoelastic material (damping $=1 \%$ ). The topographic amplifications factor in relation to the dimensionless frequency $H / \lambda$, where $H$ is the relief height and $\lambda$ is wavelength, confirms that greater amplification occurs at $H / \lambda=0.2$. This corresponds to the topographic fundamental period $T_{\mathrm{fT}}=5 H / V_{\mathrm{S}}$ of the relief. Similar values of resonance were found by Paolucci (2002); however, slightly lower values were also shown for high frequencies. In addition, in relation to the slope angle $i$, the $A_{\mathrm{T}} H / \lambda$-depending curves decrease showing greater values for $i=90^{\circ}\left(A_{\mathrm{T}} \approx 1.5\right)$, while they are lower for $i \leq 30^{\circ}$ $\left(A_{\mathrm{T}}<1.10\right)$ and negligible for $i=15^{\circ}$. Similar values were obtained for the same relief model by Nguyen et al. (2013).

In natural complex topographic zones, Maufroy et al. (2012) used a 3-D numerical simulation code in order to investigate topographic effects, in some assigned points, assuming a multi-isotropic source of seismic waves propagating in a complex 3-D media with a realistic surface topography. Their results showed topographic amplification factors up to 3.6 with a typical value range of $1.5-2.5$ at the crests. However, the 3-D topographic amplification seems to be the combined result of lithological and geometric factors in which the pure topographic effect is difficult to fully quantify in numerous cases (Gallipoli et al., 2013). In addition, in some cases, recorded ground motions show a directionality in the resonance, (Bouchon et al., 1996; Spudich et al., 1996) encountering amplification values greater than the results formulated by the 2-D and 3-D numerical simulation models (Lovati et al., 2011). Furthermore, most comparison studies refer to noise or weak aftershock motions, and thus do not take into account or only slightly take into account the non-linear effect of system ridge lithology (Gutierrez and Singh, 1992). On the other hand, the aforementioned studies have increased awareness in relation to the necessity to assess or predict topographic effect as a frequency depending variable and in an adequate way, in contrast with the simplistic models of the building codes. These models, in fact, provide the use of constant amplitudes in the entire spectrum, showing conditions of under-evaluation in several spectral ranges (Gallipoli et al., 2013; Barani et al., 2014).

\subsection{Application scenarios}

SiSeRHMap was applied to a synthetic recurrent scenario (SRS), a fictitious area of $5 \mathrm{~km}^{2}(2.5 \mathrm{~km} \times 2.0 \mathrm{~km})$, which is a synthetic reproduction of a common hilly scenario characterized by rigid/quasi-rigid reliefs and a valley with soft lithologic units covering the bedrock: the term "rigid/quasirigid" refers to the shear wave velocity values of the material constituting the relief.

The choice for using a SRS was based on the following reasons: (i) the possibility to simulate a vast number of sequences with different layer combinations in order to demonstrate the complete computational ability of SiSeRHMap; (ii) the possibility to introduce different comparison scenarios, including also real scenarios, in the analysis, as shown in the topography amplification section (Sect. 4.2). The recognizing, consultation, and interpretation of pre-existing data is a fundamental process in the definition of lithodynamic units and their spatial distribution (lithodynamic model). However, this preliminary process does not affect the performance of the code (therefore the methodology) but it affects the coherence of the results with the analysed area.

The input motion assumed in the simulation analysis is the same as that used by Grelle et al. (2014) in the real study area. It is a time-acceleration record that was spectrally matched with a general elastic spectrum design with a probabilistic target defined by the building code, which refers to a rigid site with a damping value of 0.05 . The hybrid nature of the code shows a high performance in meta-modelling when it uses an input motion with a regular (modal) acceleration response spectrum: a better performance is obtained when an input motion, matched (or fitted) in frequency with a design spectra shape (as is required in the EC8 and FEMA building codes), is given. In addition, many input motions can be inserted and processed using a partially different procedure (multi-input mode) as explained in Sect. 3.1 and 3.2. 
(a) Stratigraphic feature
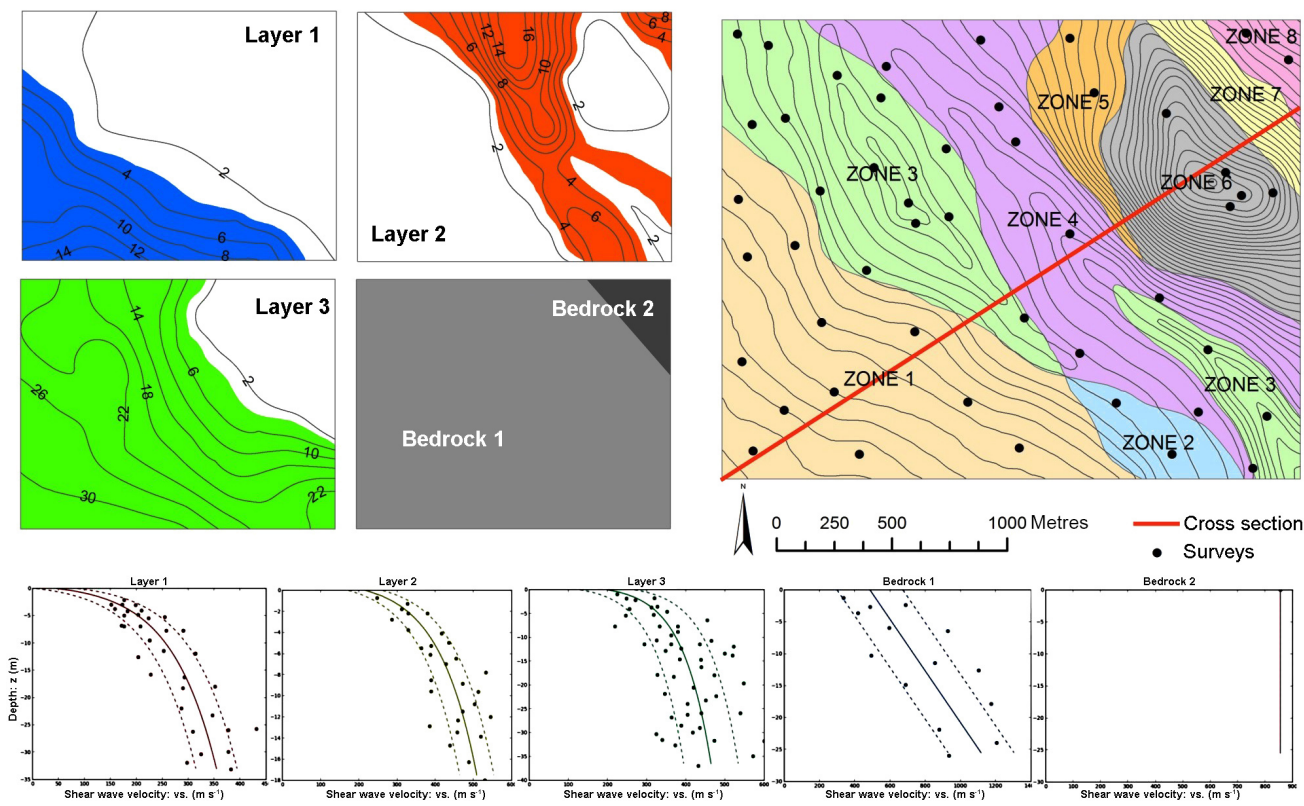

(b) Topographic feature
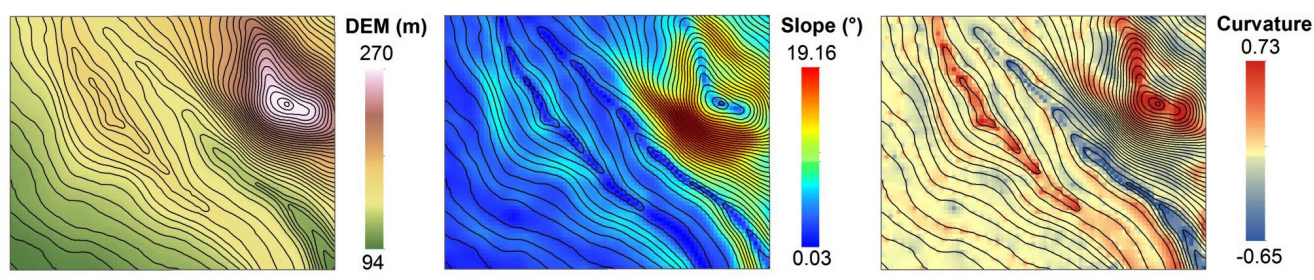

(c) Cross section

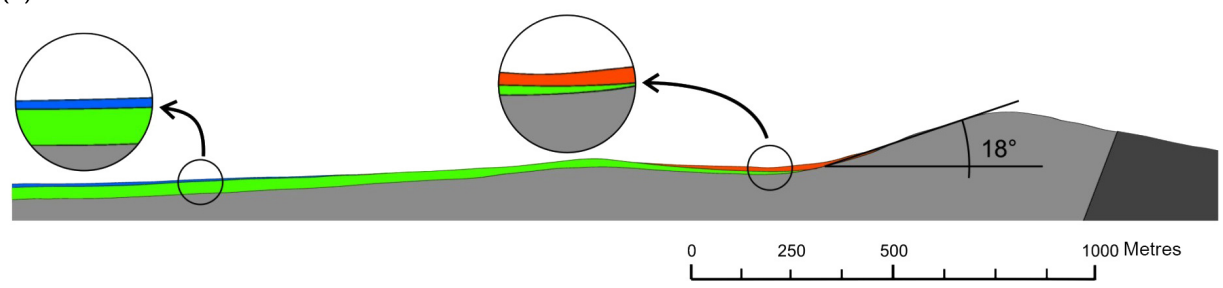

Figure 1. Synthetic recurrent scenario (SRS). (a) On the left: the maps with a resolution of $2.00 \mathrm{~m}$ regarding the covered layers and bedrock layers; for each covered layer, the iso-thicknesses of the relative lithodynamic unit, resulting from the interpolation of the hypothesized field survey is reported (black point in lithodynamic units map); the coloured polygon is the correct extension of the unit corresponding to an iso-thickness of $3.00 \mathrm{~m}$ (Sect. 2.2); on the right: the zones characterizing the synthetic recurrent scenario (SRS) are shown; (b) topographic features in terms of the DEM (digital elevation model), slope, and curvature maps with a resolution of $30 \mathrm{~m}$.; (c) cross section with zoom of the covered lithodynamic units sequence.

The stratigraphic feature of the SRS (Fig. 1a) identified three cover lithodynamic units and two bedrock, respectively, rigid and non-rigid conditions (hard rock and soft rock); with regards to the proposed methodology, the meaning of this wording will be better explained in Sect. 2. The combination of these units determines the constitution of eight zones. The number and spatial distribution of the survey points are assumed coherent in the parametric characterization, and in the geometric features of the lithodynamic units, in reference to the simple subsoil setting of the SRS. For example, if in the first analysis a lithodynamic unit is defined taking into consideration only one lithological feature, and the regression analysis does not fit well the $V_{\mathrm{S}}-z$ points distribution, it is possible to re-associate two or more lithodynamic units to the same lithology with the follow criteria: (i) clustered spatial distributions of stiffness $\left(V_{\mathrm{S}}\right)$ are recognized (horizontal accuracy) and (ii) different regression curves result as being more appropriate for characterized different depth level steps (vertical accuracy). However, in real case analyses and ignoring the ability of the modeller in the subsoil model pre- 
diction, which is based on using and/or interpreting direct or indirect survey data, the number, typology, and spatial distribution of data must be taken into account in relation to the geological complexity of the real area and the required reliability accuracy degree desired (Cardarelli et al., 2008).

The topographic feature (Fig. 1b) is characterized by a flat valley zone and a moderate high isolate relief with a slope angle of approximately $15-20^{\circ}$ and values of curvature, at the ridge, of approximately 0.5 . The resolution of the stratigraphic grid-data files and topographic grid data is different, in order to respect the resolution expected by SiSeRHMap (see Sect. 4.2). The georeferenced coordinates of the input/output grid-data files locate the SRS in southern Italy in an unreal way.

\section{GIS cubic model: $\bmod 1$ and $\bmod 2$}

The GCM (Fig. 2) is a discretized and parameterized representation of an underground half-space that is capable of performing an overlay computation of geo-referenced grid data generated by common geographic information systems platforms. This model intervenes in the SiSeRHMap in two different and non-subsequent phases. In the first phase, the model parameterizes the lithodynamic units. In the second phase, the model produces seismic response maps. The GCM structure (Grelle et al., 2014) is based on a binary template matrix in which the rows (records) and columns (fields) represent, respectively, the zones and layers.

In each zone, the presence or absence of the lithodynamic unit is defined in a binary way with attributes, respectively, value 1 and 0 . Hence, the layer, the computational entity always present in the matrix, assumes a physical entity inside it where the lithodynamic unit formalizes its presence assuming value 1 . The presence/absence of lithodynamic units is an exclusive propriety attributed to the covered layers. In contrast, the bedrock layer is always present at the base of the sequence. In this way, for a $n$-layer sequence, the maximum number of possible zones is $2^{n-1}$. The bedrock is the lithodynamic unit, which is always present at the bottom of the sequence at the $n$th layers and it can be defined as rigid or non-rigid bedrock, depending on whether the shear wave velocity is equal or greater to a prefixed threshold value, $V_{\mathrm{S}_{\text {rig }}}$; in general terms, the aforementioned bedrock typology can represent lithodynamic units composed of massive rock or weak rock. Accordingly, the term "rigid" qualifies a relative and not absolute stiffness (e.g. infinite stiffness) of the bedrock. Therefore, the condition that the non-rigid bedrock must reach the $V_{\mathrm{S}_{\text {rig }}}$ value, with depth passing thus to the rigid condition, is imposed; in this way a new lithodynamic unit up to the rigid bedrock is generated by the model; In SiSeRHMap, it is possible to consider the existence of two different bedrock typologies, thereby doubling the number of possible zones $\left(2 \times 2^{n-1}\right)$ when this occurs.

\subsection{Initial input data}

In the GCM, the number of layers, and consequently the spatial extension of the lithodynamic units, are jointly defined by preparatory studies, as is the standard procedure in high levels of seismic microzonation. These studies are based on a preliminary collection of field surveys and pre-existing studies and data sets. Subsequently, an accurate interpretation of geological, geotechnical, and geophysical data permits the definition of both the typology and characterization (parameterization), as well as the spatial distribution, of the lithodynamic units.

The main focus in the parameterization of lithodynamic units is their spatial identification; this latter can be performed taking into account the lithology and their shear wave velocity-depth value distributions. In this way, a layer is associated with each lithodynamic unit in the GCM and it is defined by a log-linear or linear depending curve, $V_{\mathrm{S}}-z$, which is identified by the intercept-velocity $V_{\mathrm{S}_{0 i}}$ and angular coefficient $\alpha_{i}$. In some cases, this identification can show how the geophysical and geotechnical proprieties of soils can be decisive in the building of a GCM model. Therefore, the equations associated with the $V_{S}-z$ lithodynamic unit distributions are

i. log-linear function for $i$ th covered layer,

$$
V_{\mathrm{S}_{i}}(z)=V_{\mathrm{S}_{0_{i}}}+\alpha_{i} \log (1+z)
$$

ii. linear function for non-rigid bedrock, $n$th layer

$$
V_{\mathrm{S}_{n}}(z)=V_{\mathrm{S}_{0_{n}}}+\alpha_{n} z ; \quad \text { where } V_{\mathrm{S}_{0_{n}}}<V_{\mathrm{S}_{\mathrm{RB}}},
$$

iii. constant value of shear wave velocity for rigid bedrock

$$
V_{\mathrm{S}_{n}}(z)=V_{\mathrm{S}_{0_{n}}} ; \quad \text { where } V_{\mathrm{S}_{0_{n}}} \geq V_{\mathrm{S}_{\mathrm{RB}}}
$$

The use of the log-linear regression function (Eq. 1) permits, in a simplified way, to also assume a uniform velocity (depth and spatial independent) for the lithodynamic units; this is possible by imposing $\alpha_{i}=0$. The log-linear law preserves the same performance of the power law equation and better robustness in the regression analysis. The linear law used for non-rigid bedrock (Eq. 2) meets the linear nature trend of the stiff soil in depth. The assumption that the uniform layers that have a progressive increase in strength and stiffness with depth is due to the increase of the effective stress and to the weakening of the material near to the surface when it is in outcropping. This assumption is well noticeable in the progressive increase of N60-SPT. Hence, taking into consideration the N60-SPT- $V_{\mathrm{S}}$ correlation equations for all soils, including stiff soils (Ohta and Goto, 1978; Imai and Tonouchi, 1982; Lum and Yam, 1994; Rollins et al., 1998), it can be seen that the non-linearity correlation occurs only with regards to low N60 values; conversely, a good linear 


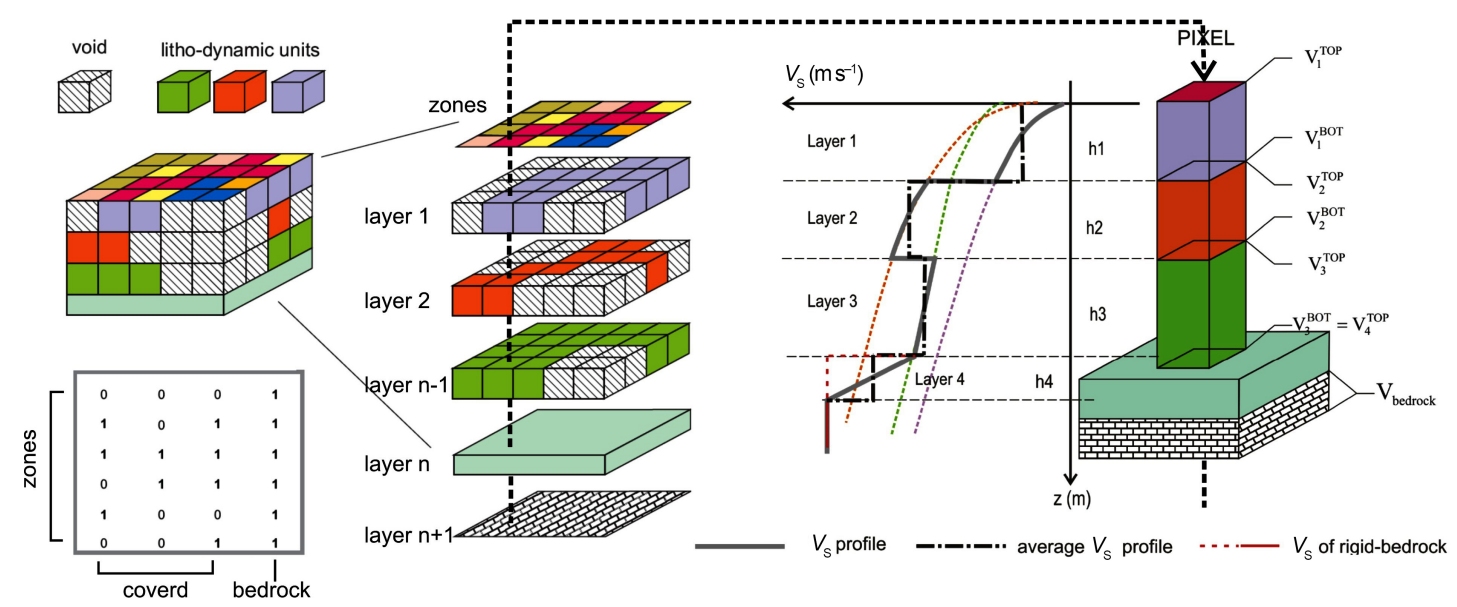

Figure 2. Subsoil half-space modelling by the GIS cubic model (GCM) and binary template matrix (e.g. referred to four layers, three covered layers, and one non-rigid bedrock) and 1-D layered $V_{\mathrm{S}}-h$ profile deriving from the GCM computational analysis (figure from Grelle et al., 2014).

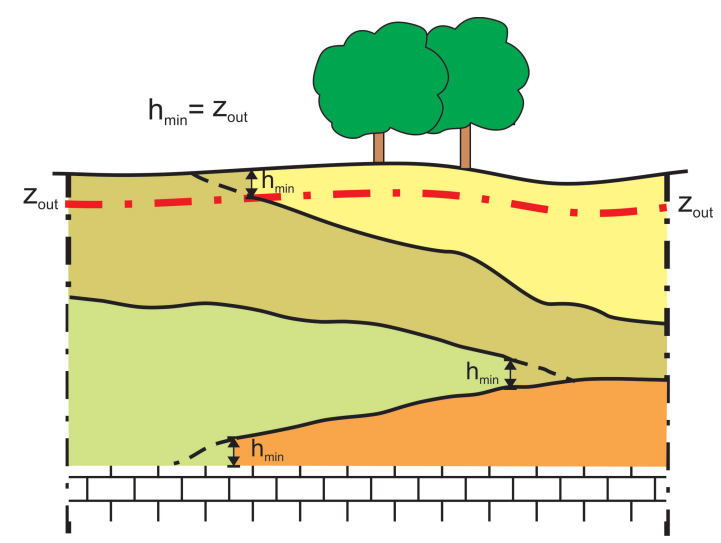

Figure 3. Example of the thicknesses cutting performed by mod2 of the SiSeRHMap.

correlation is observed for high N60 values. It is worth noting that the relation of $V_{\mathrm{S}}$ increasing with N60-SPT values is independent from the depth. Therefore, for the material constituting the non-rigid bedrock, the $V_{\mathrm{S}}$-depth linear increasing relation can be considered valid both in the buried and outcropping condition.

The curve fitting, and therefore the calibration of the parameters $V_{\mathrm{S}_{0 i}}$ and $\alpha_{i}$, are obtained by means of the leastsquares regression method (data and graphics in Supplement folder: OUTPUT \modl_VsZ).

\subsection{GCM frame}

Input grid data files containing the thickness spatial distribution of the lithodynamic units are necessary to instruct mod.2. These files are obtained via the common analysis that led to the definition of the lithodynamic units and zones. In fact, taking into consideration that the limit of a zone is also the extension line of at least one of the lithodynamic units, polyline features should define the minimum thickness as well as the borderline in the GIS pre-processing . In order to avoid computational bugs, the minimal thickness, $h_{(\min )}$, of the lithodynamic units must not be zero. More specifically, this must correspond to the depth of the output of the desired seismic response, $z_{\text {(out) }}$. Figure 3 shows how the lithology with a thickness of less than $h_{(\min )}$ did not identify the lithodynamic unit's presence; therefore, its spatial size must be preliminarily attributed to the nearest lithodynamic unit (above or below the non-identified lithodynamic unit); in 1D seismic response analysis (mod.3 Sect. 3.1), the $h_{(\min )}$ is returned in the corresponding outcropping lithodynamic unit for the computation.

In summary, the georeferenced input raster data (ASCII grid file format) are

- Layer_1.txt, Layer_2.txt, ...Layer_n-1.txt: extension of the covered layers in terms of one and zero values;

- Bedrock_1.txt, Bedrock_2.txt (if this latter is present): extension of one or two bedrock typologies in terms of one and zero values;

- Zones.txt: extension of zones that are identified from a relative integer number;

- H_layer1.txt, H_layer_2.txt, ...H_layer_n-1.txt: lithodynamic unit thicknesses obtained using appropriate GIS spatial interpolation techniques. For an adequate computational time, the grid-data resolution may be determined as follows:

top resolution unit $(\mathrm{m}) \approx$ integer $\left(\sqrt{\frac{\text { surface }\left(\mathrm{m}^{2}\right)}{10^{6}}}\right)$. 
SiSeRHMap generates new "H_layer(i)_cor.txt" files in which the thicknesses less than $h_{(\min )}$ are reported as zero. In this way, the extension of the lithodynamic units is defined in relation to the map extension of the zones. (Some grid input files are reported in the Supplement folder: INPUT $\backslash G I S \_$in.)

\subsection{GCM for $V_{S}-h$ trainer models}

Once the $V_{\mathrm{S}}-z$ curves have been obtained, and the binary template matrix has been inserted and the georeferenced grid files loaded, the GCM is thus structured and parameterized. In this phase, the GCM could start the mapped parameterization of the shear wave velocity for each layer as reported in Grelle et al. (2014). However, in SiSeRHMap this computational process is performed in a subsequent second phase of the GCM (mod.5). In this first phase, the GCM gives data regarding the thicknesses range of the lithodynamic units in the zones to obtain the appropriate $V_{\mathrm{S}}-h$ trainer models reproducing the 1-D subsoil models as selected in a randomly uniform way in the GCM. Therefore, the nature of the methodology requires that the equations, which characterize and parameterize the GCM, are equal to those that will be used in the generation of the $V_{\mathrm{S}}-h$ trainer models; thus, these equations will be subsequently circumstantiated at a generic $(x, y)$ geographic point, in the second phase of the GCM (GCM maps executor).

The $V_{\mathrm{S}}-h$ trainer models (Fig. 4) are defined by the subsequent equations (5 to 10 ) using the thickness values extracted, from the uniformly random distribution (Monte Carlo technique), within the maximum and minimum intervals found for each lithodynamic unit in each zone. The number of the models generated is freely chosen but it should be assumed taking into account thickness variability and the number of the lithodynamic units present in the zones (the default value is 10).

Therefore, once the GCM has been structured according to a $(m \times n)$ binary template matrix and the $q$ number of the $V_{S^{-}}$ $h$ trainer models has been established, mod.2 of SiSeRHMap generates the $V_{\mathrm{S}}-h$ trainer models. In this way, the parameterization of an $i$ th layer $(i$ in $[1, n])$ in a $j$ th zone $(j$ in $[1, m])$ for a $k$ th $V_{\mathrm{S}}-h$ trainer model $(k$ in $[1, q])$ are defined by the following points.

i. The shear-wave velocity at the top and bottom of each $n-1$ cover layer is obtained using the parameterized log-linear functions; in relation to the combining of the layers position, the inversion of shear rigidity is also possible.

$$
\begin{aligned}
& V_{\mathrm{S}_{i(j, k) \text { top }}}=V_{\mathrm{S}_{0_{i}}}+\alpha_{i}\left\{\log \left[1+\left(\sum_{i=1}^{n-1} h_{i-1(j, k)}\right)\right]\right\} \\
& V_{\mathrm{S}_{i(j, k) \text { bot }}}=V_{\mathrm{S}_{0_{i}}}+\alpha_{i}\left\{\log \left[1+\left(\sum_{i=1}^{n-1} h_{i(j, k)}\right)\right]\right\}
\end{aligned}
$$

ii. With regards to the rigid bedrock, $V_{\mathrm{S}_{\mathrm{rig}}}$, it is defined in relation to an established threshold of the shear wave velocity (e.g. $V_{\mathrm{S}_{\text {rig }}} \geq 800 \mathrm{~m} \mathrm{~s}^{-1}$, EC8 prEN1998). In this way, the rigid bedrock is defined by a unique value of the shear-wave velocity $V_{\mathrm{S}_{\mathrm{RB}}}$ with the condition that $V_{\mathrm{S}_{\mathrm{RB}}} \geq V_{\mathrm{S}_{\mathrm{rig}}}$.

In contrast, when the bedrock is non-rigid (geological bedrock), the GCM automatically generates a new layer with a thickness of $h_{n(x, y)}$ and it assumes the $n$th position while the rigid bedrock layer shifts to the $(n+1)$ th position. The latter layer has a lithodynamic nature similar to non-rigid bedrock but its depth confers to it the characteristics of rigid bedrock with a shear wave velocity equal to $V_{\mathrm{S}_{\mathrm{RB}}}$. This condition is defined by the following equation:

$V_{\mathrm{S}_{n(j, k) \text { bot }}}=V_{\mathrm{S}_{\mathrm{RB}}}$

thus it results that

$h_{n(j, k)}=\frac{\left(V_{\mathrm{S}_{\mathrm{RB}}}-V_{\mathrm{S}_{n(j, k) \mathrm{top}}}\right)}{\alpha_{n}}$,

where

$V_{\mathrm{S}_{n(j, k) \text { top }}}=\max \left(V_{\mathrm{S}_{n-1(j, k)} \text { bop }} ; V_{\mathrm{S}_{0 n}},\right)$

$\alpha_{n}$ is the gradient, and the $V_{\mathrm{S}_{0 n}}$ is the intercept value relating to the $V_{\mathrm{S}}$-depth regression linear curve of the non-rigid bedrock (Eq. 2). In Eq. (8), when the max value is $V_{\mathrm{S}_{n-1} \text { Bot }}$, the possible increment of rigidity due to the lithostatic load of the upper cover layers is taken into account; this case is manifested when the non-rigid bedrock shows relatively low values of the shear wave velocity in the spatial statistical uncertainty of the $V_{\mathrm{S}}, z$ values. In contrast, when the max value is $V_{\mathrm{S}_{0 n}}$, this indicates that the non-rigid bedrock is near to the rigid condition and therefore it shows relatively high values of the shear wave velocity in the $V_{\mathrm{S}}-z$ dispersion curve.

iii. The average shear-wave velocity of each lithodynamic unit is

$\bar{V}_{\mathrm{S}_{i(j, k)}}=\frac{1}{2}\left(V_{\mathrm{S}_{i(j, k) \mathrm{top}}}+V_{\mathrm{S}_{i(j, k)} \mathrm{bot}}\right)$.

iv. The fundamental vibration period computed considering the average shear wave velocity obtained using the average travel time:

$$
T_{f_{(j, k)}}=\frac{4 \sum_{i=1}^{n} h_{i(j, k)}}{\sum_{i=1}^{n} h_{i(j, k)} / \sum_{i=1}^{n}\left(h_{i(j, k)} / \bar{V}_{\mathrm{S}_{i(j, k)}}\right)} .
$$

When the training model is composed only of the rigid bedrock (outcropping rock), the value of $T_{\mathrm{f}}$ is assumed to be $0.01 \mathrm{~s}$. 

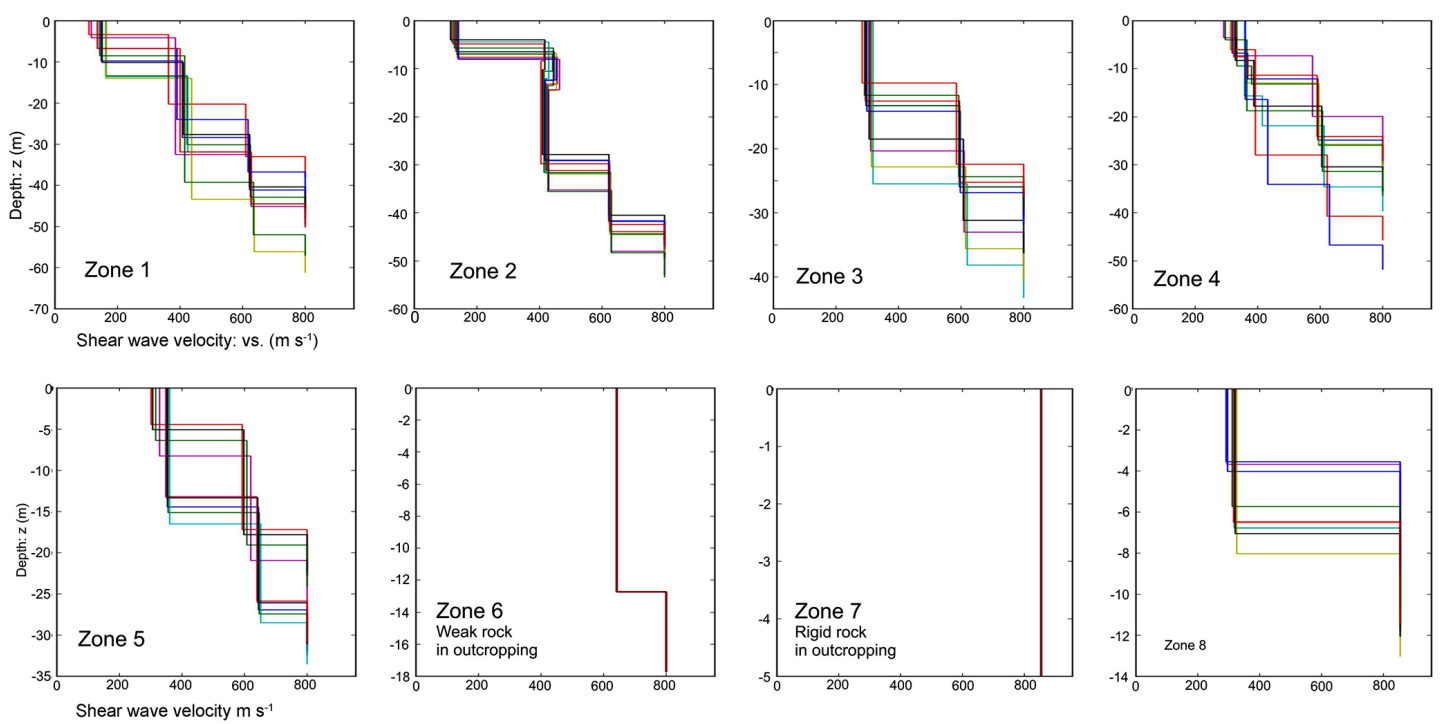

Figure 4. $V_{\mathrm{S}}-h$ trainer models: there are 10 trainer models theoretically encountered in each of the eight zones, which are presented in the SRS (Fig. 1a).

\section{Meta-modelling: $\bmod 3$ and $\bmod 4$}

The meta-model process is the core of SiSeRHMap. This process is composed of a semi-automated computation of the stratigraphic seismic responses of the $V_{\mathrm{S}}-h$ trainer models selected. Subsequently, a new robust and performing prediction model "Emul-spectra" is trained on the spectral shape of these responses in order to emulate the stratigraphic seismic response in the succeeding GCM maps executor (mod.5).

\subsection{Stratigraphic seismic response}

The stratigraphic acceleration response spectra is performed in SiSeRHMap by mod.3: stratigraphic response. Here, the dynamic site response is computed in a similar way to other computer program/codes: SHAKE (Schnabel et al., 1972; Idriss and Sun, 1992; Ordónez, 2009), EERA (Bardet et al., 2000), and STRATA (Kottke and Rathje, 2008, 2010). The module computes the dynamic acceleration response that refers to a 1-D soil column using a planar vertical wave propagation model, which takes into consideration an equivalent shear-strain-dependent dynamic response of the soil sequence. This method is commonly referred to as the viscoelastic equivalent linear analysis, in terms of total stress, taking into consideration a linear elastic bedrock. A horizontal polarized propagation of the shear waves through a site with infinite horizontal layers is assumed (Appendix A).

Despite the same computational performance of similar software (Fig. 5), mod.3 is dedicated to processing uploaded data from previous modules and subsequently returns data that are used in the next computational module (mod.4). Specifically, the Stratigraphic Seismic Response module performs an automatic computation of all the selected $V_{\mathrm{S}}-h$ trainer models. The natural unit weight, $\rho$, associated with each layering profile is empirically estimated in relation to the shear wave velocity. In this way, taking into account the low influence of this variable on the shear modulus due to its limited variation, the natural unit weight can be defined (Keçeli, 2012) as

$\rho=4.4 V_{\mathrm{S}}^{0.25}$,

where $\rho$ is expressed in $\mathrm{kN} \mathrm{m}^{-3}$.

The input motion is considered on the outcropping to the rigid rock. Therefore, it is always deconvoluted within the sequence on the rigid bedrock (layer $n$ or $n+1$ ), when the covered layers are present in the zone. The output response (Fig. 6) is provided at the outcropping of the surface detected by the assigned $z_{\text {out }}$ depth; this surface is within the upper layer.

For each covered lithodynamic unit, as well as the nonrigid bedrock, the initial damping ratio, such as the straindependent values of the normalized shear module and the damping ratio, must be inserted. From these latter values, the damping ratio and shear modulus degradation curves are obtained using the regression analysis in the $G(\gamma) / G_{0}$ and $D(\gamma)$ ratio curves fitting, which was introduced by Yokota et al. (1981) (Appendix A). Therefore, the computational iteration permits a convergence of both the equivalent calculated strain, $\gamma_{\mathrm{eq}}=\left(r \cdot \gamma_{\max }\right)$, and the trial strain, where $\gamma_{\max }$ is the maximum strain encountered in the dynamic time history, while $r$ is the strain equivalent ratio; this can be freely assigned (the default value is 0.65 ) or it can be estimated in relation to an assigned earthquake magnitude, $M$, by the equation:

$r=\frac{M-1}{10}$. 


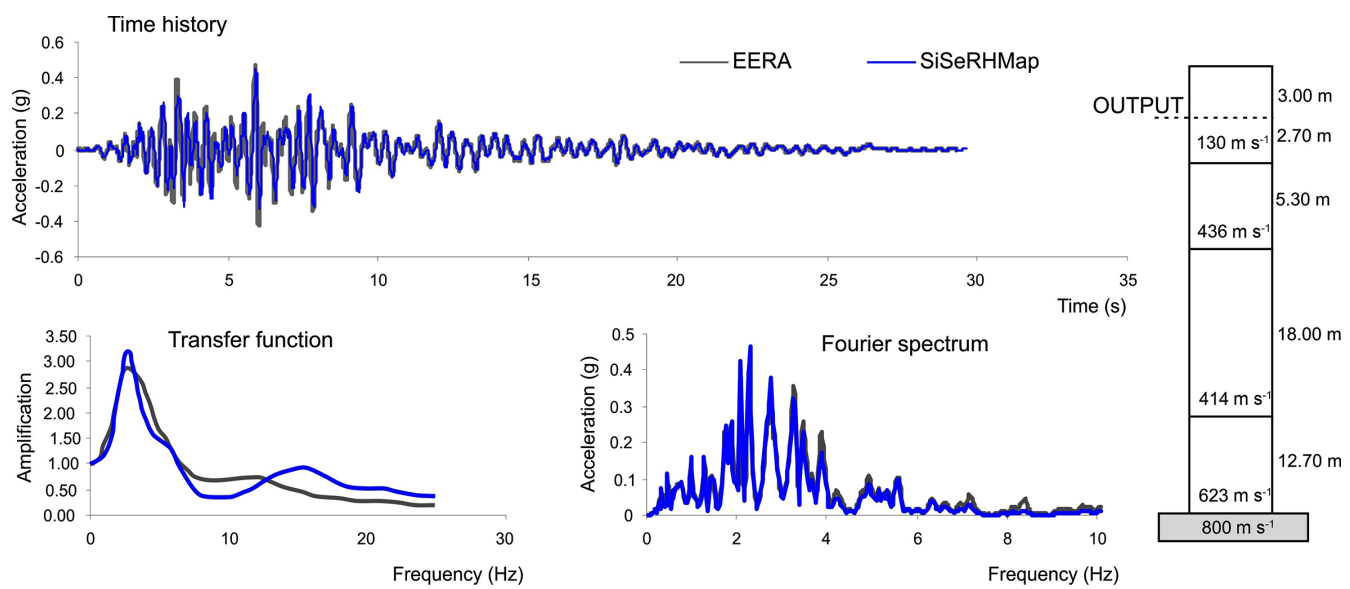

Figure 5. Comparison between EERA and SiSeRHMap (mod.3, stratigraphic response) on a 1-D model related to the third trainer $V_{\mathrm{S}}-h$ model regarding zone 2 .

A number of iterations of 5-10 largely assures the convergence of a dynamic solution (the default value is: 10); in contrast the use of a number of iterations equal to zero entails a pure viscoelastic linear analysis. Nonetheless, a constant value of the damping ratio is assumed for rigid bedrock. This value is attributed both to the fixed rigid bedrock and to the rigid bedrock resulting from non-rigid bedrock (the default value is: 0.01 ). For the zones characterized by outcropping rigid rock, the seismic response is automatically referred to the input motion.

The aforementioned process can be iterated using more assigned input motions; in this case the code is able to generate the average seismic responses constituting the training models used in the following meta-modelling process. However, the smoothed responses, generated by the trained meta-model, suggest a better performance for input motions with the acceleration response spectra nearest, or matched, to the simplified code design spectra. On this subject, the multi-input motion mode performs the stratigraphic seismic response analysis for each input motion on all the $V_{\mathrm{S}}-h$ selected profiles in a separate way. Therefore, average acceleration response spectra are obtained from a set of output acceleration response spectra computed for each zone; these average spectra are the trainer models used in the subsequent meta-model procedure. However, it is worth noting, as previously stated, that better performances of the meta-model are given using input motions that provide an average response spectra matched (or fitted) on the design code spectra shape (a complete example is illustrated in Fig. 8).

In the stratigraphic response module, an additional module "view signal" (Fig. 6) is associated in order to plot the time history signal (acceleration and strain) and spectra (transfer function, Fourier spectra, response spectra). (Some input and output files are reported in the Supplement folders: INPUT \Dynamic_properties; OUTPUT \mod3_Seismic_Response.)

\section{2 "Emul-spectra": adaptive simulation model}

Emul-spectra, $\Psi$, are a numerical adaptive model capable of emulating the theoretical stratigraphic seismic response. In this way, this model assumes a key role promoting the hybrid evolution of the procedures in SiSeRHMap.

The Emul-spectra model is introduced here and it stems from the previous experience of Grelle et al. (2014) in which hypotheses relating to the behaviour assumed by combinations of multi-parametric functions were introduced with the aim of obtaining good performances in the fitting of the acceleration response spectra. In Emul-spectra, the natural influence on the spectral-trends of some main physical parameters are largely taken into consideration, confirming previous studies regarding principal component analysis (PCA). The physical parameters used as independent variables in Emulspectra are (i) the average shear wave velocity of the nearsurface lithodynamic unit, $V_{\mathrm{S}(\mathrm{up})}$; (ii) the elastic fundamental period of the sequence, $T_{\mathrm{f}}$, and (iii) the period, $T$. Its analytical form is

$$
\begin{aligned}
\Psi= & \frac{x_{1}}{V_{\mathrm{S}(\text { up })}\left(1+x_{2} T^{2}\right)} \\
& +K \frac{x_{3}^{T_{\mathrm{f}} \log \left(V_{\mathrm{S}(\mathrm{up})}\right)}}{\exp \left[\left(x_{4} T_{\mathrm{f}}+x_{5} T\right)^{2}\right]\left(T_{\mathrm{f}}+x_{6} T\right)^{x_{7} \frac{T_{\mathrm{f}}}{\log \left(V_{\mathrm{S}(\mathrm{up}))}\right.}}} \\
& \log \left(1+T^{2}\right)+x_{8} \frac{T_{\mathrm{f}}}{T V_{\mathrm{S}(\text { up })}^{2}},
\end{aligned}
$$

where $x_{1}, \ldots, x_{8}$ are the eight calibration parameters (coefficients) and $K$ is the modal scaling factor. Emul-spectra permit a unique solution for each zone; in this way, the parameter, $T$, can be considered a fast-changing variable (spectral variable), whereas the $V_{\mathrm{S} \text { (up) }}$ and $T_{\mathrm{f}}$ change in relation to the $V_{\mathrm{S}}-h$ profile model (local variables) and the aforementioned eight calibration parameters are constant coefficients (zone variables). For zones with rigid rock outcrops, $T_{\mathrm{f}}$ assumes a 


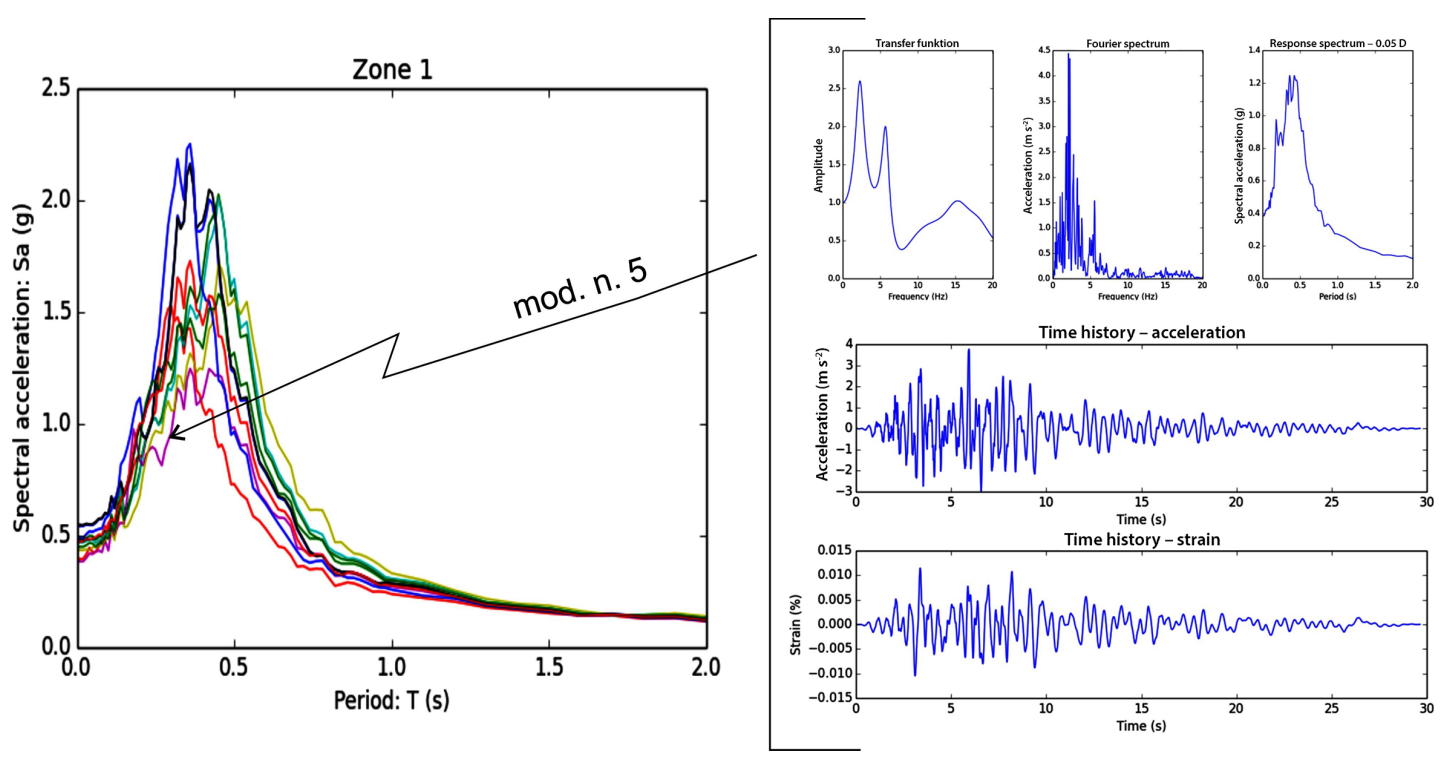

Figure 6. Example of the Stratigraphic seismic response set of zone 1 with 0.05 damping; for this set, the graphics plotted of the signal view module related to the fifth trainer $V_{\mathrm{S}}-h$ model are also shown. In the analysis (all zones), the equivalent stress ratio is obtained by Eq. (13), taking into consideration a magnitude of 6.4 .

value of $0.01 \mathrm{~s}$ and the $V_{\mathrm{S}(\mathrm{up})}$ is set equal to the corresponding rigid bedrock.

The three component functions, summed to define Emulspectra (Eq. 14), have specific and different roles in the fitness performance of the model. To this regard, and considering $\Psi$ as being dependant on $T$, it is worth highlighting that (i) the first component has the role of "bed function" because it is the platform of the other component functions due to the fact that it greatly controls the intercept at the zero-period (Peak Ground Acceleration, PGA) and the tail fitting values; (ii) the second component is the "modal function" that controls the fitting peak values in the modal shape; and (iii) the third component is the "PGA-correction function", which corrects the initial values permitting a more accurate fitting of the PGAs. In the bed function, the intercept (PGA) is inversely dependent on $V_{\mathrm{S}(u p)}$, although an addition or subtraction that is sign $x_{8}$-coefficient dependent, is specifically performed by the PGA-correction function. The latter, in relation to the trend shown between $T_{\mathrm{f}}$ and PGA in the seismic response of a specific zone, permits taking into account the possible known non-linear effect to decrement the spectral values at high frequencies (low periods). The modal function is the core of the Emul-spectra adaptive model. It is a exponential equation capable of reproducing a symmetrical/asymmetrical modal or subordinated bimodal shapes generally shown by acceleration seismic responses in a large spectral range (e.g. in Fig. 7), as well as in the multiinput probabilistic way (Fig. 8). The modal function, which combines the parameters $V_{\mathrm{S}(\mathrm{up})}$ and $T_{\mathrm{f}}$ in a different way, permits a chasing of the various peak-trend distributions by zones as well as possible single spectral behaviours or possi- ble non-peak-trend conditions due to the different influences of the non-linear responses. The modal scaling factor, $K$, acts only on the modal function. It is usually assumed to be equal to 1.00 and can be changed after calibration in order to scale the peaks.

In mod.4 of SiSeRHMap, Emul-spectra are trained on the theoretical spectra response values (mod.3), which are sampled starting from an initial period value of $0.001 \mathrm{~s}$ (PGA) and continue with regular sampling within the chosen spectral interval. The initial period value is fixed, while the sample rate (the default value is $0.1 \mathrm{~s}$ ) and the number of samples (the default value is 15), and therefore the spectral interval, can be introduced by the operator. The choice of the aforementioned values is fundamental since these define the efficacy and congruence of the meta-model. In addition, the window sampling establishes the periods for which the seismic response maps will be returned which, in turn, will influence the design spectral maps. Taking into account that the sampling interval is equal for all the zones, this should include the whole spectral energy part without exceeding in the sampling of the spectral tail. In fact, the performance of fitness on the energy spectral part can be weak when a high number of tail values is involved. The training of Emul-spectra aim at finding the optimized solution for the eight calibration parameters (Appendix B). It is performed by a nearing solution process by means of a dedicated evolutionary algorithm (EA) and a final optimizer algorithm: the Levenberg-Marquardt algorithm (LMA). The latter is a curve-fitting algorithm used in many software applications for solving generic inverse problems. 
(a)

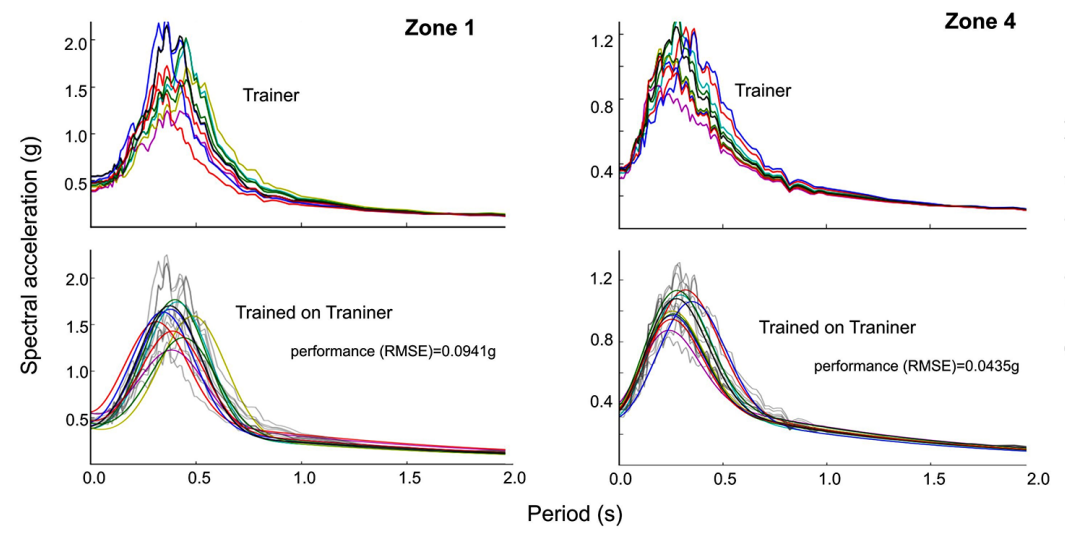

(b)

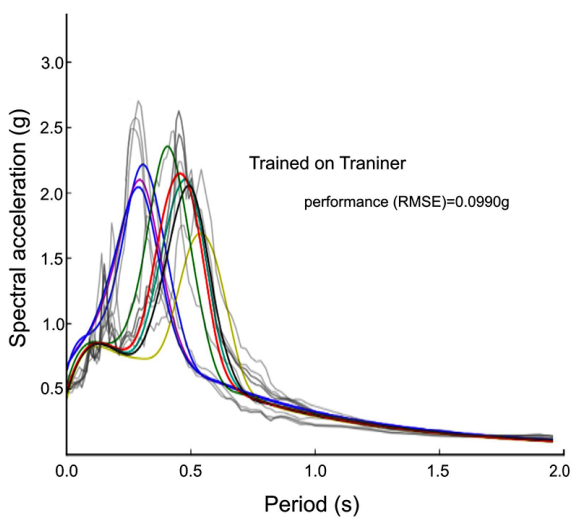

Figure 7. Performance of Emul-spectra: (a) stratigraphic seismic response with a damping of 0.05 regarding some trainer $V_{\mathrm{S}}-h$ profiles of the SRS (all graphics are reported in the Supplement). The resulting performance defined by RMSE (g) are zone 1=0.0941; zone $2=0.0862$; zone $3=0.0544$; zone $4=0.0435$; zone $5=0.0370$; zone 6 (non rigid rock in outcropping) $=0.0032$; zone 7 (rigid rock in outcropping $=0.0045$; zone $8=0.0394$ (b) example on stratigraphic seismic responses that show a large spectral variability; the trainer spectra are obtained by the notable increasing of the top-layer thicknesses in the zone 1 models.

The EA is a meta-heuristic method based on an evolutionary elitism of the offspring solutions that mutate up to satisfying or converging into a predefined fitness condition. The fitness of the solutions is defined by the fitting error, which is expressed in terms of a mean square error (MSE). The EA is constituted by two breeding levels. In the first level, the offspring solutions are generated according to a corresponding Gaussian distribution in which the mean values representing the initial guesses population (low range parental) and corresponding standard deviations are supplied. In an iterative way, in the first level, only the population of offspring solutions, which shows a fitness better than the previously encountered solutions, is allowed to pass to the second level in accordance with the elitism process. The number of procreations is four (fixed) and for each successive generation the probable parental affinity is increased (Appendix B). The elitism process is reset (mass extinction) when an assigned number of population solutions is reached and the convergence has not been reached yet. The convergence event occurs when an incremented assigned initial (minimum) error target $E_{\text {targ }}$ is found. This error is increased by a assigned ratio (the default value is 0.01 ) at the end of the second breeding level when the process returns to the first breeding level. The assigned value of the initial error target depends on the shape of the training seismic response curves in reference to the shape ability of the Emul-spectra model. However, the fitting, and consequently the $E_{\text {targ }}$ value, can be dependent on the number of the randomly selected models, $\mathrm{Nm}$, and on the number of the lithodynamic units present in the sequence, Nl. Taking this aspect into account, the default values of $E_{\mathrm{targ}}$ are empirically defined, for each zone, as follows:

$E_{\mathrm{targ}}=\frac{(\mathrm{Nm} \times \mathrm{Nl})}{1000}$.
The choice of an appropriate $E_{\text {targ }}$ avoids a long computational time or, in contrast, the occurrence of premature convergences.

Optionally, in the meta-model module $(\bmod 4)$, it is possible to select the zone where an additional computation of "refinement" can be performed. This re-processing may be run when the fit or the shape regression curves are not considered satisfactory by the operator. The new processing can be performed using the initial guess parameters obtained in the previous processing and new standard deviation values, as well as a new lower $E_{\text {targ }}$, can be assigned.

\section{GCM maps executor: $\bmod .5$}

The maps executor is the second phase of the GCM and the last module of SiSeRHMap. In this phase, the GCM module generates the hybrid stratigraphic seismic response maps (Fig. 9) after having further parameterized the model using data developed by the previous modules and some new inserted data. Therefore, a hybrid seismic response (HSR) can be computed both in reference only to the stratigraphic seismic response or also taking into account the topographic amplification effect. Data in relation to the latter are computed by an ancillary sub-module: "topographic amplification" that requires new geo-referenced topographic data files. Finally, an additional ancillary sub-module, the "design spectra", permits the computation of the damped synthetic design response spectra that envelopes the seismic response spectra using the composed functions with shapes in accordance with EC8 and FEMA. (Some grid output files are reported in the Supplement folder: OUTPUT \GIS_out .) 

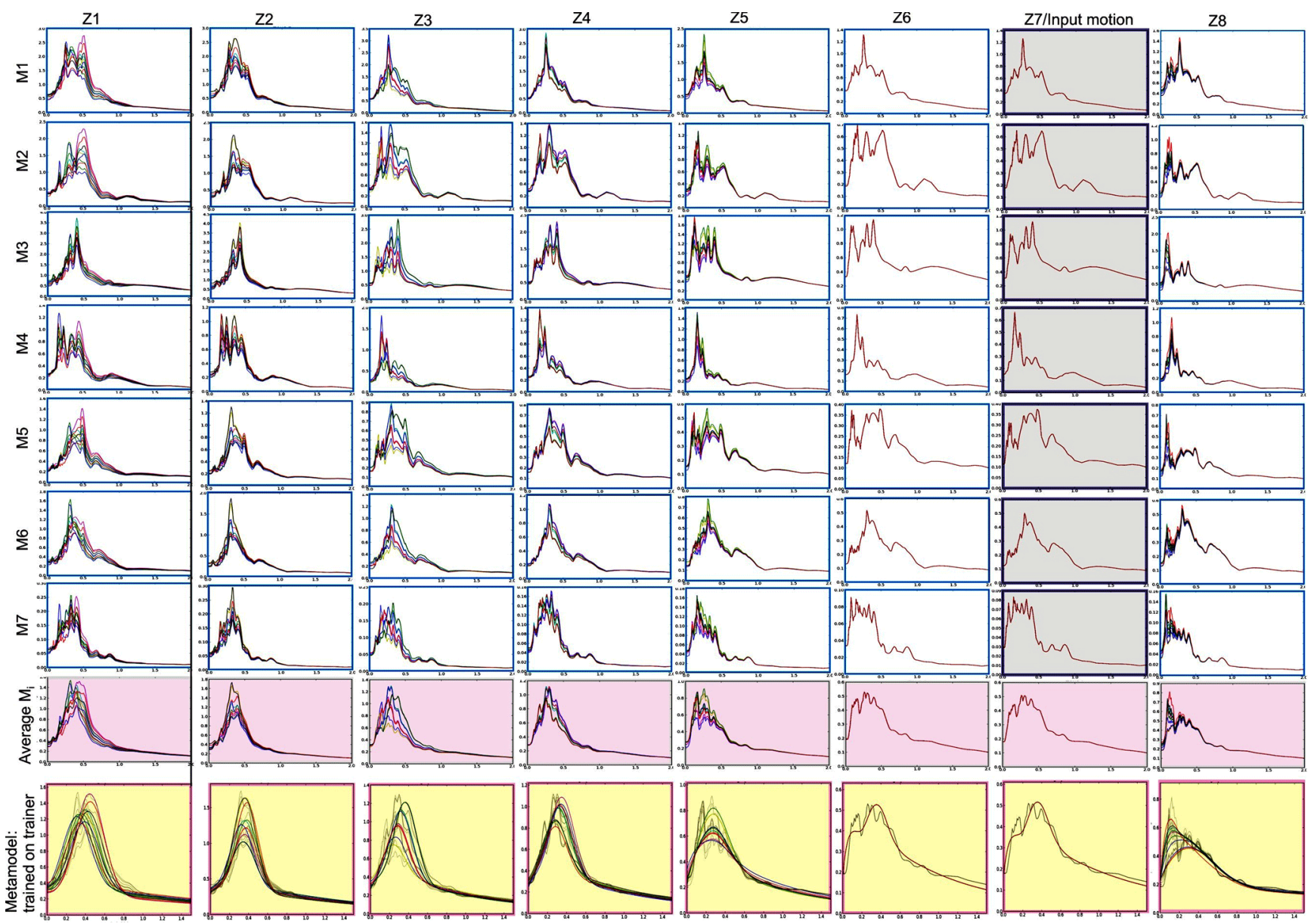

Figure 8. Example of meta-model processing for the SRS using seven input motions having average spectrum matched on an unamplified design spectrum. This last corresponding to the average spectrum of the zone $\mathrm{Z7}$ where the rigid rock outcrops.

\subsection{Stratigraphic seismic response mapping}

For every geographic $x, y$ point, the GCM is able to associate a corresponding $j$-zone and consequently also the relative parameters, processes, and information deriving from the previous modules. In this second phase, the GCM proceeds to configure itself using the common physic bases and hypothesis assumed in the construction and parameterization of the trainer $V_{\mathrm{S}}-h$ profiles (Sect. 2.3). These are as follows:

i. The average shear wave velocity, $\bar{V}_{S_{i(x, y)}}$, of the lithodynamic units, which is computed in accordance with Eq. (10); it assumes a value of zero where the lithodynamic is not present in the layer. In addition, if nonrigid bedrock is present at the bed of the sequence, the GCM generates the $n$-cover layer in which the $h_{n(x, y)}$ and $V_{\mathrm{S}_{n(x, y)}}$ are defined in accordance with Eq. (9).

ii. The fundamental period $T_{\mathrm{f}_{(x, y)}}$ is computed in accordance with Eq. (11). In addition, where the rock is outcropped, the fundamental period assumes a value of $0.01 \mathrm{~s}$. iii. In each zone, the GCM recognizes the average shear wave velocity of the nearest surface lithodynamic unit $V_{\mathrm{S}_{\mathrm{up}(x, y)}}$.

Once the GCM is parameterized, it is able to define the hybrid stratigraphic seismic response (Fig. 8) by solving the numerical model Emul-spectra (Eq. 14) that in this context assumes the form:

$$
\Sigma(T)_{(x, y)}=f\left[(T),\left(V_{\mathrm{S}_{\mathrm{up}(x, y)}}, T_{0_{(x, y)}}\right),\left(\left(x_{1}\right)_{j} \ldots\left(x_{8}\right)_{j}\right)\right],
$$

where the period $T$ assumes the values in the spectra interval for which Emul-spectra have been trained. The GCM maps executor computes the hybrid seismic response using the same period used in the meta-model training.

The maps of hybrid stratigraphic response (Fig. 9) can be affected by a quick change of data near the border of the zones; this effect can be due to the different fitting performed by the meta-model calibration as well as the geometrical cutting of the thickness discussed in Sect. 2.2. In order to take into account these affects, SiSeRHMap permits the use of spatial Gaussian smoothing. 

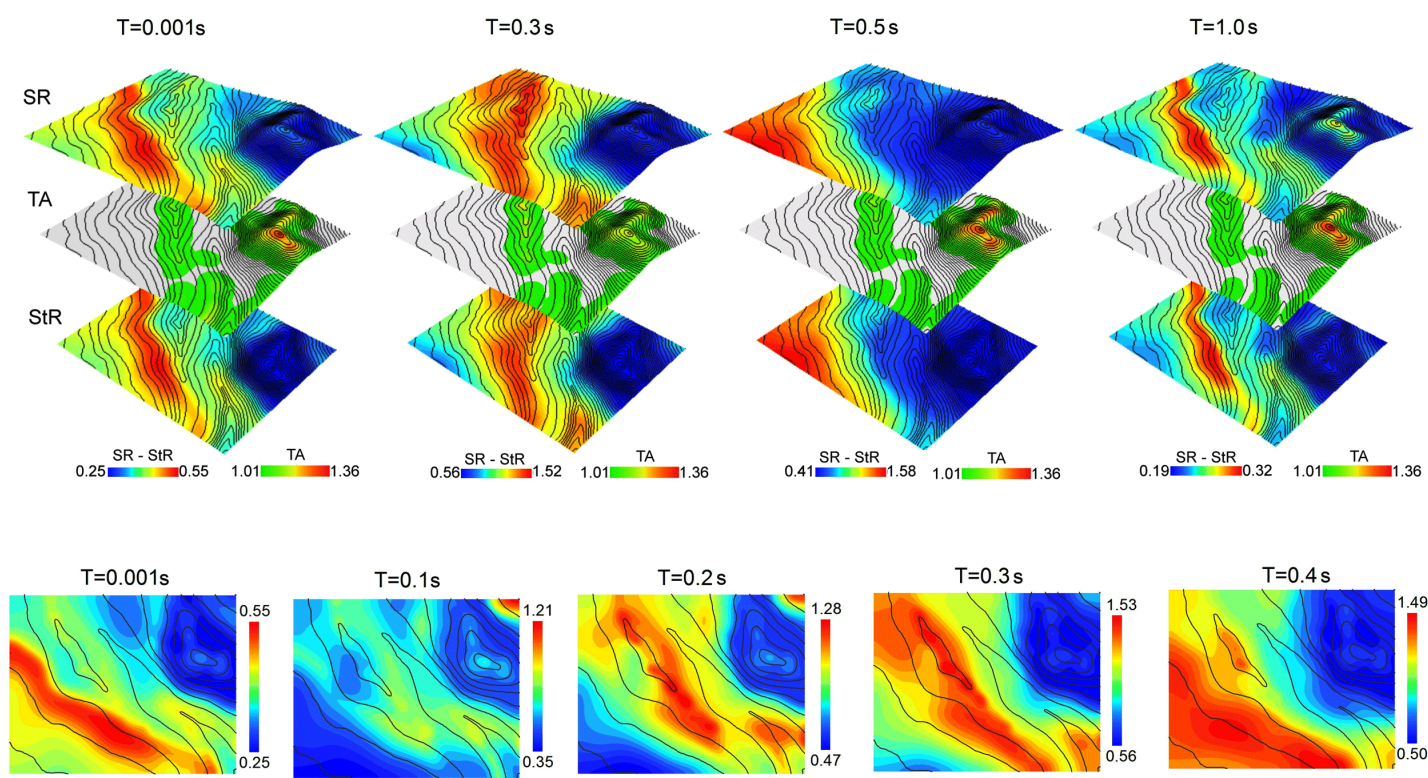

$\mathrm{T}=0.1 \mathrm{~s}$

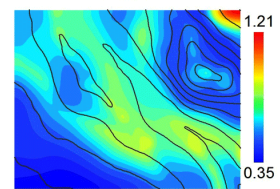

$\mathrm{T}=0.2 \mathrm{~s}$
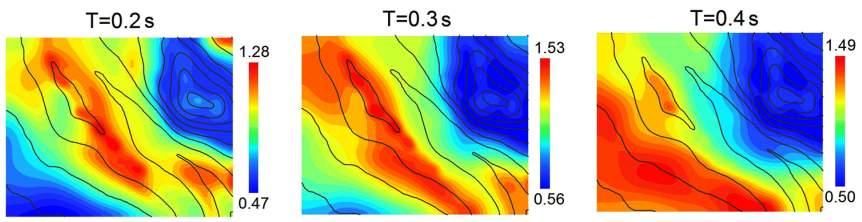

$\mathrm{T}=0.5 \mathrm{~s}$
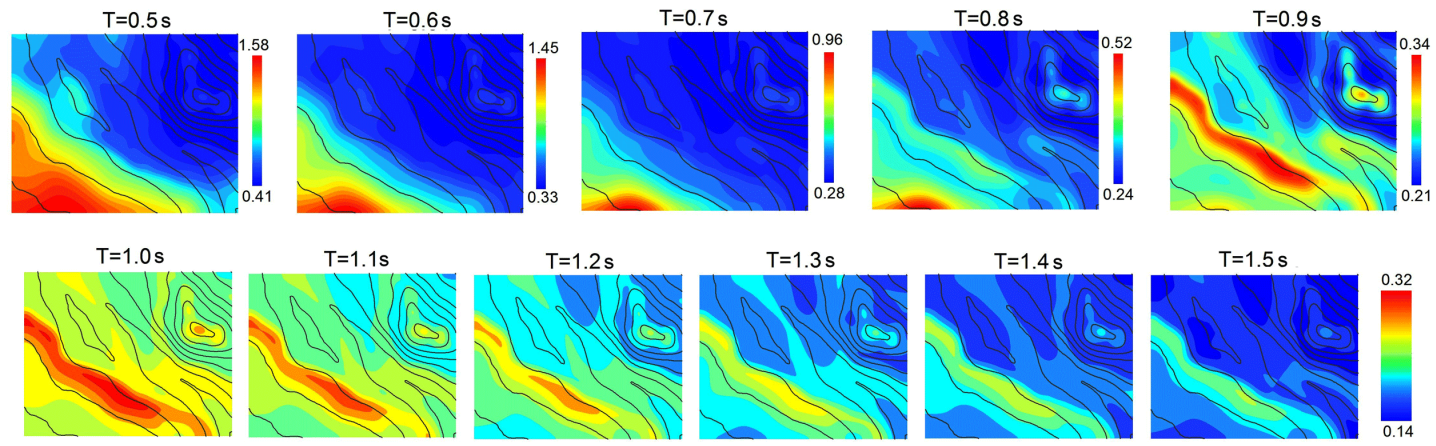

Figure 9. Set of seismic response maps for different periods. The combined effect of the stratigraphic and topographic features are shown at the top of the figure; StR is the stratigraphic seismic response, TA is the topographic amplification and SR is the seismic response.

\subsection{Topographic amplification mapping}

A prediction model has been developed based on pre-existing studies and simulations on the effects of topographic amplification on seismic motion (Geli et al., 1988; Ashford et al., 1997; Maufroy et al., 2012, 2015). This model, trained on 2-D regular reliefs and balanced on 3-D landforms, aims at predicting the spatial amplification effect on the seismic response of reliefs, considering them to be constituted by homogeneous material. To this scope, digital topographic attributes are used to introduce morphometric variables into the model. These are (i) digital elevation model, DEM (DTM_30.txt); (ii) slope angle, $i$ (slope_30.txt), which is the arctangent of the first derivate of the DEM; and (iii) curvature, $c$ (curvature_30.txt), which is the second derivative of the DEM. The latter is the inverse of the ray curvature, which is expressed in terms of a resolution unit ratio. Therefore, a positive value of the curvature represents convex features, such as ridges or edges, while a negative value indicates concave features, such as a valley. A geometric trend of the curvature and slope along a typical profile relief (the upper part of Fig. 10) illustrates that the curvature assumes a greater value on the ridge, where the slope is minimum or near to zero, and the curvature assumes a zero value where the slope angle is greater. Towards the valley, the slope angle decreases while the curvature assumes negative values down to the minimum. The curvature is expressed in terms of the maximum values in relation to the 3-D minimum curvature radius, which implicates that the topographic amplification model tends to predict the maximum amplification associated with the transversal polarized motion of the relief.

On the aforementioned bases, the prediction model of topographic amplification is a spatial-frequency dependent model constituted by a combination of the two sub-models (the lower part of Fig. 10). Taking into account a generic $(x, y)$ point, $A_{\mathrm{Tc}}$ is the prediction model for the topographic amplification in ridge/edge regions:

$$
A_{\mathrm{Tc}}=1+c \eta_{t} e^{-2 \eta_{t}}+A_{1} c \eta_{t}^{2} e^{-A_{2} \eta_{t}^{2}}+A_{3} \eta_{t} e^{\sqrt{c}}
$$




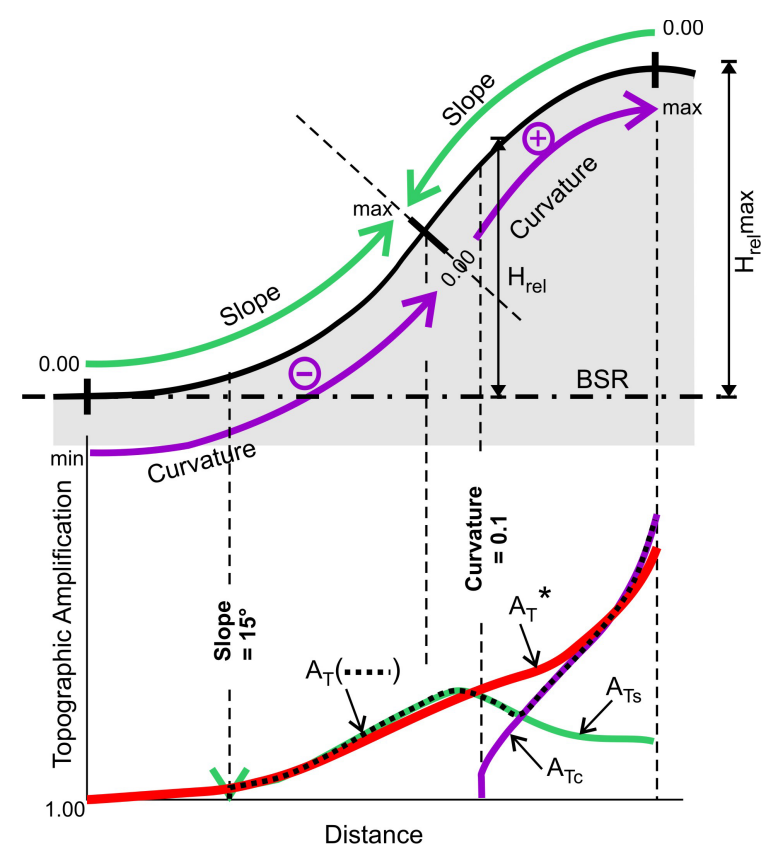

Figure 10. The behaviour components of the topographic amplification model in relation to the distribution of the GIS-topographic attributes (DEM, slope, and curvature) along an isolated half-relief.

and $A_{\mathrm{Ts}}$ is the prediction model for the topographic amplification along the slope surface:

$$
\begin{aligned}
A_{\mathrm{Ts}}= & +\left\{r _ { H } \left[\left(1+\frac{B_{1} c}{2 \sqrt{\pi}} e^{-B_{2} \eta_{t}^{2}(1+c)}+B_{3} \log \eta_{t}\right)\right.\right. \\
& \left.\left.\left(1+\sin ^{2} i\right)\right]-r_{H}\right\},
\end{aligned}
$$

where $r_{H}=H / H_{\mathrm{R}}$ and it is the relief ratio in which $H$ and $H_{\mathrm{R}}$ are, respectively, the local slope height and the relief height, both of which are taken into consideration by the basal surface of relief (BSR) where $H=0 . A_{1}, A_{2}, A_{3}$ and $B_{1}, B_{2}, B_{3}$ are the calibration parameters defined on the results obtained by the numerical model analysis of the 2-D homogeneous relief (discussed below in this section); for Eq. (17), a subsequent light calibration on real 3-D cases (Sect. 4.2.1) is also affected. Hence, the dimensionless frequency, defined as slope height/wavelength, is

$$
\eta_{t}=\frac{H}{V_{\mathrm{S}_{\mathrm{Reg}}} T},
$$

where the $V_{\mathrm{S}_{\mathrm{Reg}}}$ is the regional shear wave velocity. Finally, the topographic amplification $A_{\mathrm{T}}$ is the maximum value of $A_{\mathrm{Tc}}$ and $A_{\mathrm{Ts}}$ for each $(x, y)$ point.

SiSeRHMap permits the definition of the BSR in relation to features of the topographic area (Appendix C), while the regional shear wave velocity must be assigned. This represents the average shear wave velocity of the rigid material constituting the relief(s), that can be different (frequently greater) to the shear wave velocity of the rigid bedrock assumed in the stratigraphic response analysis. Thus, in SiSeRHMap, the topographic sub-module permits the simulation of the 3-D surface amplification mainly on the basis of morphometric data and using an assigned uniform stiffness of the reliefs with the task of shifting the frequency distribution of the amplification data.

In general terms, the behaviour of the $A_{\mathrm{Tc}}$ and the $A_{\mathrm{Ts}}$ depends on the curvature and on the slope angle topography attributes, which, in turn, depend on the value of the spatial resolution unit as well as the elevation resolution (sampling altitude value). In order to take into account these conditions, the prediction models are calibrated on grid curvature data related to the spatial resolution unit of $30 \mathrm{~m}$, which can be 1 order of magnitude higher than the resolution unit of the stratigraphic response (Eq. 4). In order to meet this assumption, a specific computational algorithm within the method excludes the natural ripples of the slope, which can be confused with ridges; in addition, the aforementioned assumption is sustained by the fact that the amplification of low rigid ridges (less than $30 \mathrm{~m}$ in height) occurs in frequencies that usually have very little effect on buildings. The algorithm necessitates a recognition of the complete topographic features of the region that is the subject of the stratigraphic response analysis; in some cases, this aspect involves taking into consideration an area much larger than one object of the stratigraphic response analysis. Subsequently, the algorithm performs an extracting, a georeferencing, and a resolution adaptation to the smaller target area that corresponds to the stratigraphic response area. In addition, the output grid-maps are Gaussian smoothed using a calibrated standard deviation value (expressed in the number of the resolution units) depending on the elevation resolution previous used for the development of the input topographic attribute maps. The calibration function derives from a sensibility analysis based on the invariant of the output data.

The $A_{\mathrm{Tc}}$ and $A_{\mathrm{Ts}}$ prediction models (Eqs. 17 and 18) are devised in a frequency dependant manner and calibrated in amplitude taking into account the findings and results derived from several simulation analyses based on physical models. Therefore, from these latter, the following calibration parameters (Eqs. 17 and 18) result as being $A_{1}=90$, $A_{2}=30, A_{3}=0.25$ and $B_{1}=3.60, B_{2}=3.24, B_{3}=0.12$. With regards to the modelling and calibration of $A_{\mathrm{T}}$, Fig. 11 shows a geometrical model, similar to that considered by Geli et al. (1988), with a typical shape of the isolate relief of a middle-high altitude area (hilly area). In this setting, a curvature of 0.5 is associated with the ridge, while the maximum of the slope angle of $30^{\circ}$ is reached at the mid-point of the relief. As illustrated, the topographic prediction models are nevertheless devised to provide amplified or non-amplified responses; consequently, they do not include spectral deamplification (predominant in the valley), but they provide the peak values near to the topographic fundamental period of the relief. In addition, the $A_{\mathrm{Tc}}$ model provides the peak 
and it is predominant on the curvature zone (e.g. ridge or topographic border), while the $A_{\mathrm{Ts}}$ model is predominant along the slope, as expected. This last model defines the amplification curve for high periods, in all the cases.

For some corresponding positions along the surface of the relief, the comparison with the numerical simulation performed by Geli et al. (1988) shows (Fig. 11) that the topographic prediction model, $A_{\mathrm{T}}$, is able to perform an adequate and efficient overlap, such as in comparison to the topographic edge feature (Ashford et al., 1997). An application in real areas (Fig. 12) illustrates the performance and the ability of the code to resolve the topographic model, by way of a preliminary definition of the BSR and the relief ratio, $r_{\mathrm{H}}$. The mapping restitution process provides for a computational optimization, mainly aimed at minimizing the unreasonable concentration of high values. These high values are caused by natural roughness, in addition to an anomaly in the base-digital map. The computational optimization, of $A_{\mathrm{T}}$ in $A_{\mathrm{T}}{ }^{*}$, consists in the smoothed numerical bass cut of the slope angle $<15^{\circ}$, curvature $>0.1$, and $H_{\mathrm{R}}<30 \mathrm{~m}$.

The simplified frequency-dependent topographic amplification model, reported in Eqs. (17) and (18), is mainly focused on the peak/ridge amplification effect (position 1 in Fig. 10) that is the greatest effect in the relief. The prediction accuracy on the slopes is the result of the progressive spatial smoothing of the topographic amplification and the conservative approach, too. The latter does not admit deamplification. Diversely, it admits a suitable overmatch (overestimation) in almost the entire spectral window. In this way, it gives the possibility to preserve an adequate prediction trend for irregular reliefs too. This aspect should be seen in light of the fact that the values of the slope topographic amplifications are generally lower than those that occur in the peak zones.

\subsubsection{Validation}

Differently to the meta-model process at the base of the stratigraphic seismic response, the topographic model may not be trained on local specified cases of theoretical effects. The topographic model is based on surface 3-D-depending variables (DEM, slope, and curvature) that define the shape of relief(s) and, in general, of the terrain conformation. Therefore, this model was built and calibrated in order to take into account substantial case studies of hilly mountain sceneries which are prone or susceptible to seismic topographic effects.

Bearing in mind that the strong natural spatial changing of topographic attributes influences the efficacy of the topographic amplification model of SiSeRHMap, some validation tests were performed on real areas in order to verify the accuracy and robustness of its predictions. Two real hilly mountain areas were selected due to (1) their setting diversity and (2) the availability of in depth analysis, in terms of experimental characterizations and numerical simulations, car- ried out by other authors. The comparison cases (Fig. 13) regard: (i) the Albion Plateau area (France) (Maufroy et al., 2012, 2015) - a topographically articulate area constituted by hilly reliefs with complex shapes and with different directions of their stretching axis; and (ii) the Narni relief (Italy) - a well-defined and partially isolated asymmetric relief, approximately $1300 \mathrm{~m}$ long and with variable heights and basal widths.

In the first case (Fig. 13a), a 3-D numerical simulation of the topographic amplification was performed on the central part (target area) of the Albion Plateau area where 200 random double-couple point sources (fault plains modelling) were considered at approximately $4 \mathrm{~km}$ depth, in a homogeneous subsoil half-space. In this way, waves with different incidences and intensities were contemplated. The simulation analysis was performed using a 3-D partly staggered finite difference code (Cruz-Atienza 2006). Moreover, the elastic and isotropic subsoil medium was modelled with shear and compression wave velocities of 3000 and $5000 \mathrm{~m} \mathrm{~s}^{-1}$, and a density of $2.6 \mathrm{~g} \mathrm{~cm}^{-3}$. Specifications on the processing modality and parameterization are reported in Maufroy et al. (2012). The comparison in the frequency domain was performed in terms of wave lengths in different representative points regarding different topographic real features. The points and the chosen frequency are identical to those reported in Maufroy et al. (2015).

The results provided by the topographic model in SiSeRHMap demonstrate how its predicted horizontal spectral amplifications are mainly included between the 50th and 84th percentile of the amplification values resulting from a numerical multi-source simulation for each of the five cases (Fig. 13a). In addition, it should be noted that the spectral peaks match the tendency of the numerical simulation. The matching is more evident in the ridge of the relief where the topographic amplification is greater; the deamplification effects shown in the slope perched valley and bottom valley are predicted as a non-amplification effect in observance of the nature and the character of the proposed model.

The second case (Fig. 13b) takes into consideration the seismic data recorded by means of temporary seismometric stations installed in correspondence to the ridge (set of seven stations) and the base (set of three stations) of the Narni hill, in the period of March-September 2009, intercepting the L'Aquila seismic sequence. In this period, 702 earthquake events were recorded, of which 12 with $M_{\mathrm{L}}>4.0$. Details regarding the used sensors and the recording procedure are reported in Massa et al. (2012). The analysis regards the experimental methods in seismic response estimation in order to characterize the topographic spectral amplification effect. The spectral amplification ratio (SSR) and the Horizontal to Vertical Spectral Ratio (HVSR) procedure were computed in Massa et al. (2012); the SSRs results, defined in terms of average and standard deviation values, are reported in Barani et al. (2014) where data of 2-D numerical simulations are also reported in the same terms. This numerical analysis re- 

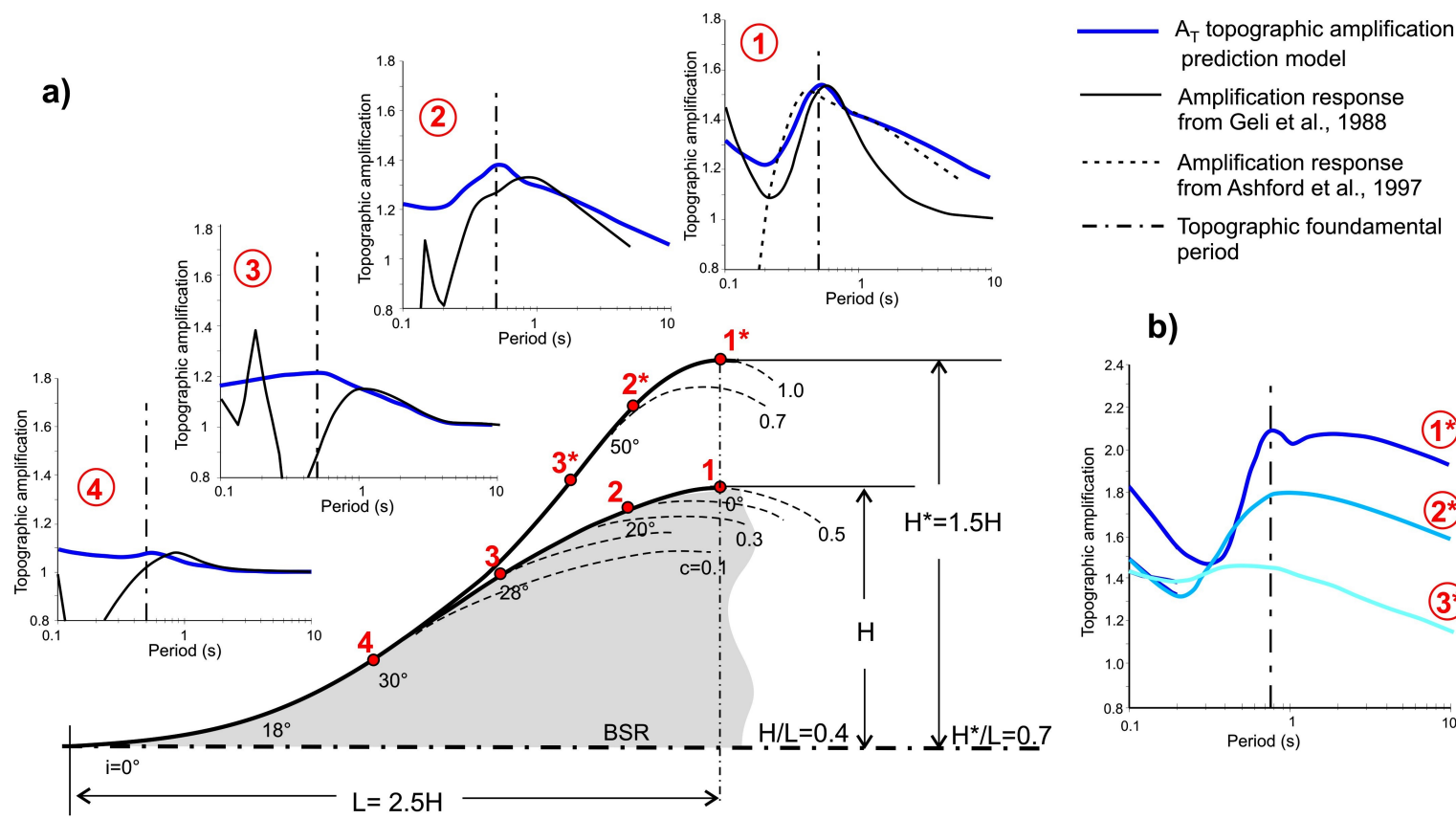

b)
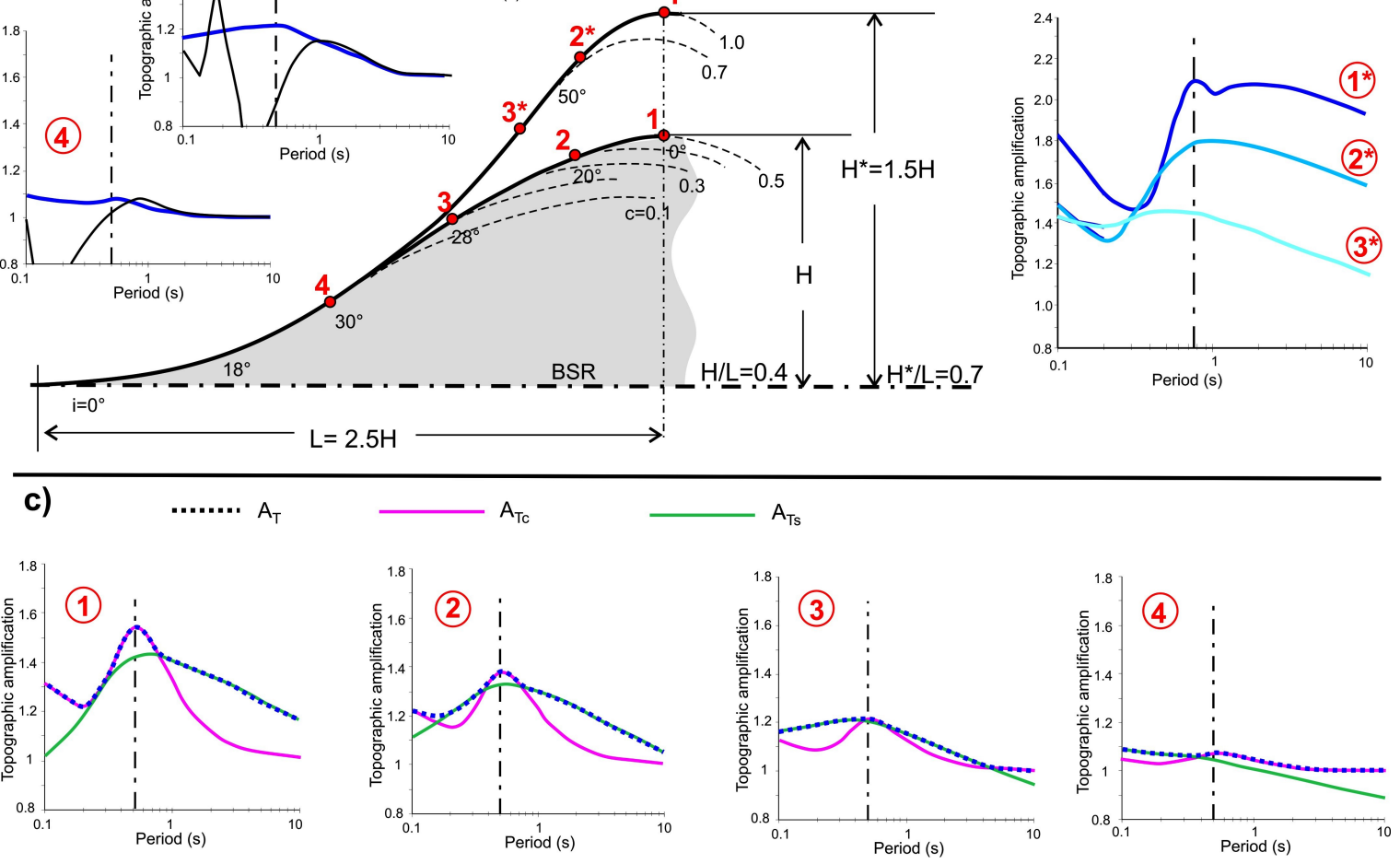

Figure 11. Performance of the topographic prediction model, $A_{\mathrm{T}}$, along an isolate half-relief; this is similar to that used in the numerical simulation by Geli et al. (1988). (a) The simulation considers vertical incident SH waves; in the same way, the Ashford et al. (1997) simulation analysis regards the ridge of the relief with a slope angle of $90^{\circ}$; (b) topographic prediction projected on a more pronounced relief; (c) topographic prediction model $A_{\mathrm{T}}$ illustrated in term of combined shape of $A_{\mathrm{Tc}}$ and $A_{\mathrm{Ts}}$ models. The topographic fundamental periods is corresponding to $H / \lambda=0.2$ (Geli et al., 1988 .

gards two simplified geometrical models characterized by a uniform relief with $V_{\mathrm{S}}=1400 \mathrm{~m} \mathrm{~s}^{-1}$, a double layer relief with $V_{\mathrm{S}}=2000 \mathrm{~m} \mathrm{~s}^{-1}$ for the outcropping top layer, and $V_{\mathrm{S}}=1400 \mathrm{~m} \mathrm{~s}^{-1}$ for the bed layer; the same authors report that the relief rock material is constituted by massive limestone with diffused fracture patterns at the near surface. Considering these models, two regional shear wave velocities, $V_{\mathrm{S}_{\mathrm{Reg}}}$, of 1500 and $2000 \mathrm{~m} \mathrm{~s}^{-1}$, were used for the simulation by the topographic model of SiSeRHMap. The results show a migration to high frequency that occurs when the regional shear velocity increases; this effect appears less evident for the peak that protrudes on the plain (3-D shape). The topographic computing module of SiSeRHMap was applied on an area that includes approximately $1500 \mathrm{~m}$ of the relief's length. However, the comparison was focalized on the first part, at approximately $700 \mathrm{~m}$ of the protruding area, where experimental and numerical simulation data were available in order to perform the validation analysis. The extraction of the 2-D spectral amplification factor along the edge and ridge of the relief highlights the 3-D nature in the prediction analysis of the model. On this subject, the local saddle feature (in $B$ and $B^{*}$ ) along the ridge conserves high amplification values on the edge and a substantial decreasing at the central ridge (crest), reported in Fig. 13b.

The comparison analysis takes into consideration the topographic amplification distribution along the ridge profile, obtained assuming a $V_{\mathrm{S}_{\mathrm{Reg}}}$ of $2000 \mathrm{~m} \mathrm{~s}^{-1}$ in the proposed topographic model; this value seems to provide the best match with the experimental data. It is worth noting that there is an agreement in frequency ( 3 to $5-6 \mathrm{~Hz}$ ) between the average spatial distribution horizontal amplification developed by SiSeRHMap and the non-directional and direc- 


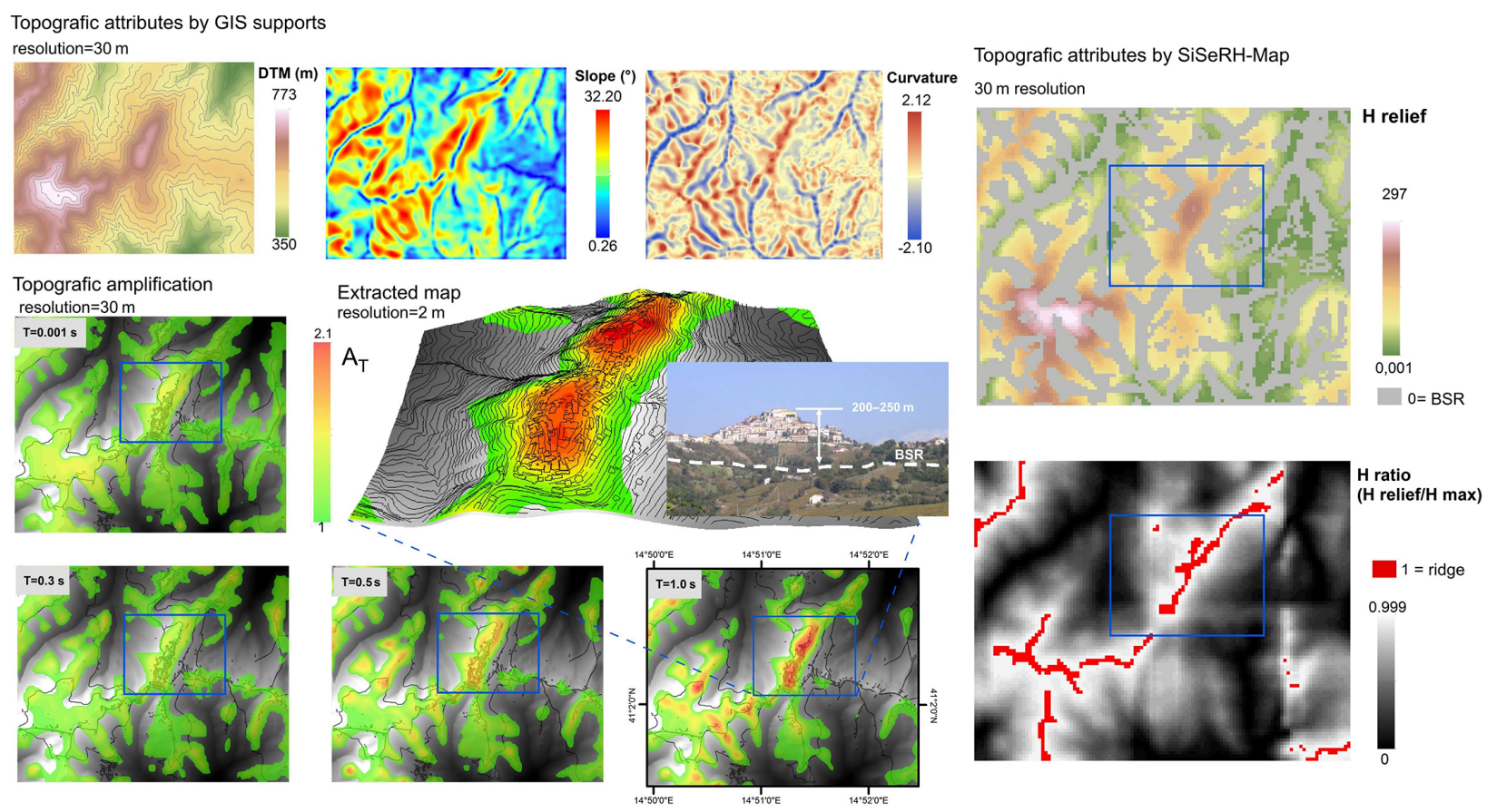

Figure 12. Example of topographic amplification computed on a real hill-mountain area of southern Italy: blue box is the automatic splitting map of the urbanized area of the village of Montefusco.

tional (transversal to relief) horizontal amplification of the average SSRs values in the zone subject to seismic stations at the top of the relief. The 2-D numerical simulation, with an amplification from 5 to $8 \mathrm{~Hz}$, does not match the SSR values. With regards to the amplification results, they show spectral average values slightly greater by a factor of up to 3 and 4 for non-directional SSRs, respectively, and up to 2 for the topographic prediction model in SiSeRHMap; this last factor is also shown in the 2-D numerical analysis. On this specific topic and in agreement with scientists working on this area (Lovati et al., 2011; Massa et al., 2012; Barani et al., 2014), it is possible to hypothesize a net overlapping spectra between the stratigraphic and the topographic effects. In support of the aforementioned effects, the spectral amplification results obtained by the HVSR analysis and the non-directional SSRs intervene showing peaks of fundamental periods ( 3 to $5 \mathrm{~Hz}$ ) close to the directional SSRs values. A more detailed debate on this topic is reported in the discussion paragraph.

\subsection{Design spectra mapping}

The design spectra are obtained by the envelopment of the HSR in observance of the synthetic spectra drawn by the discontinuous function, which defines the elastic response in Euro Code 8 as well as in FEMA 356 (2000). The envelope technique used here needs to take into account the discrete nature of the hybrid seismic response. The technique (Fig. 14) consists in the following computational steps: i. recognition of the period, $T_{\mathrm{p}}$, showing the maximum value (peak) of the hybrid seismic response $\mathrm{HSR}_{\max }$;

ii. computation of the mean, $M$, of the HSR values, which are greater than the intercept $\mathrm{HSR}_{0}$ value at period $T=$ $0.001(\approx \mathrm{PGA})$

iii. computation of the mean $M_{\mathrm{R}}$ and $M_{\mathrm{L}}$ of HSR values greater than $M$, respectively, to the right and left of $\mathrm{HSR}_{\max }$;

iv. in this way the characterized parameters of the design spectra are

$$
\begin{aligned}
a_{0} & =\mathrm{HSR}_{0}, \\
f_{0} & =\frac{\mathrm{HSR}_{\max }}{\mathrm{HSR}_{0}}, \\
T_{B} & =T_{\mathrm{p}}\left[1-\left(\frac{M}{M_{\mathrm{L}}} \frac{N_{\mathrm{L}}}{N}\right)\right], \\
T_{C} & =T_{\mathrm{p}}\left[1+\left(\frac{M}{M_{\mathrm{R}}} \frac{N_{\mathrm{R}}}{N}\right)\right], \\
T_{D} & =1.6+\left(4 \mathrm{HSR}_{0}\right),
\end{aligned}
$$

where the $N=\left(N_{\mathrm{L}}+N_{\mathrm{R}}\right)$ is the number of HSR values over the $M$, and $N_{\mathrm{L}}$ and $N_{\mathrm{R}}$ are the respective numbers of the values to the left and right, excluding the $\mathrm{HSR}_{\max }$, in counting. 
(a) Albion Plateau area (France)
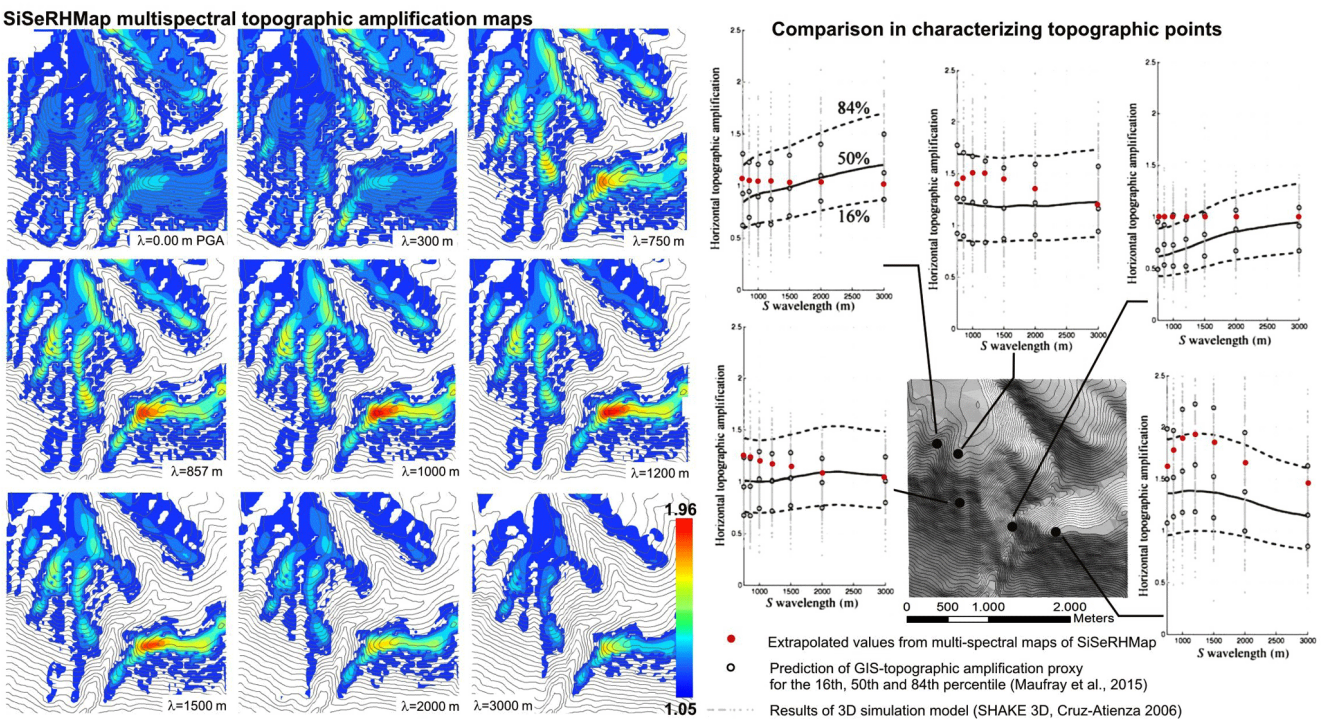

(b) Narni prominent hill (Italy)
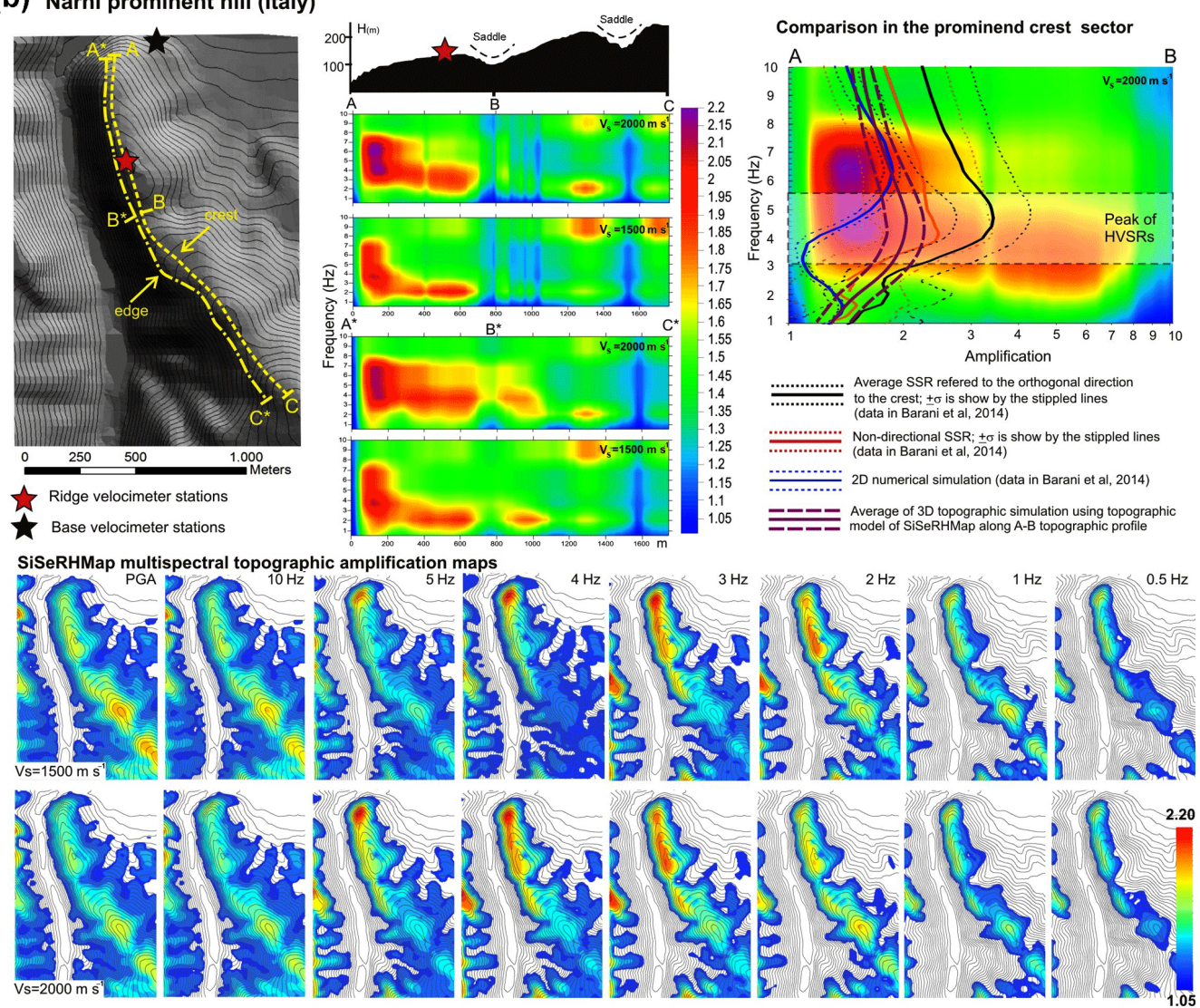

Figure 13. (a) Albino Plateau area (France): SiSeRHMap multi-spectral topographic amplification maps shown in terms of wavelength, $\lambda$, assuming a $V_{\mathrm{S}}$ of $3000 \mathrm{~m} \mathrm{~s}^{-1}$ and using a resolution in elevation of $20 \mathrm{~m}$. Comparison in characterizing topographic points between the map-extrapolated values and the results of 3-D simulation model (SHAKE 3D; Cruz-Atienza 2006); results of GIS-topographic amplification proxy, which is built and calibrated in this specific area (Maufroy et al., 2015). (b) Narni prominent hill (Italy): SiSeRHMap multi-spectral topographic amplification maps defined assuming $V_{\mathrm{S}}$ of 1500 and $2000 \mathrm{~m} \mathrm{~s}^{-1}$; performance of the model along the edge and crest profile. Comparison analysis referring to a sector of the crest profile $(A-B)$ with results of the experimental and 2-D numerical simulation model (Massa et al., 2010; Barani et al., 2014). 

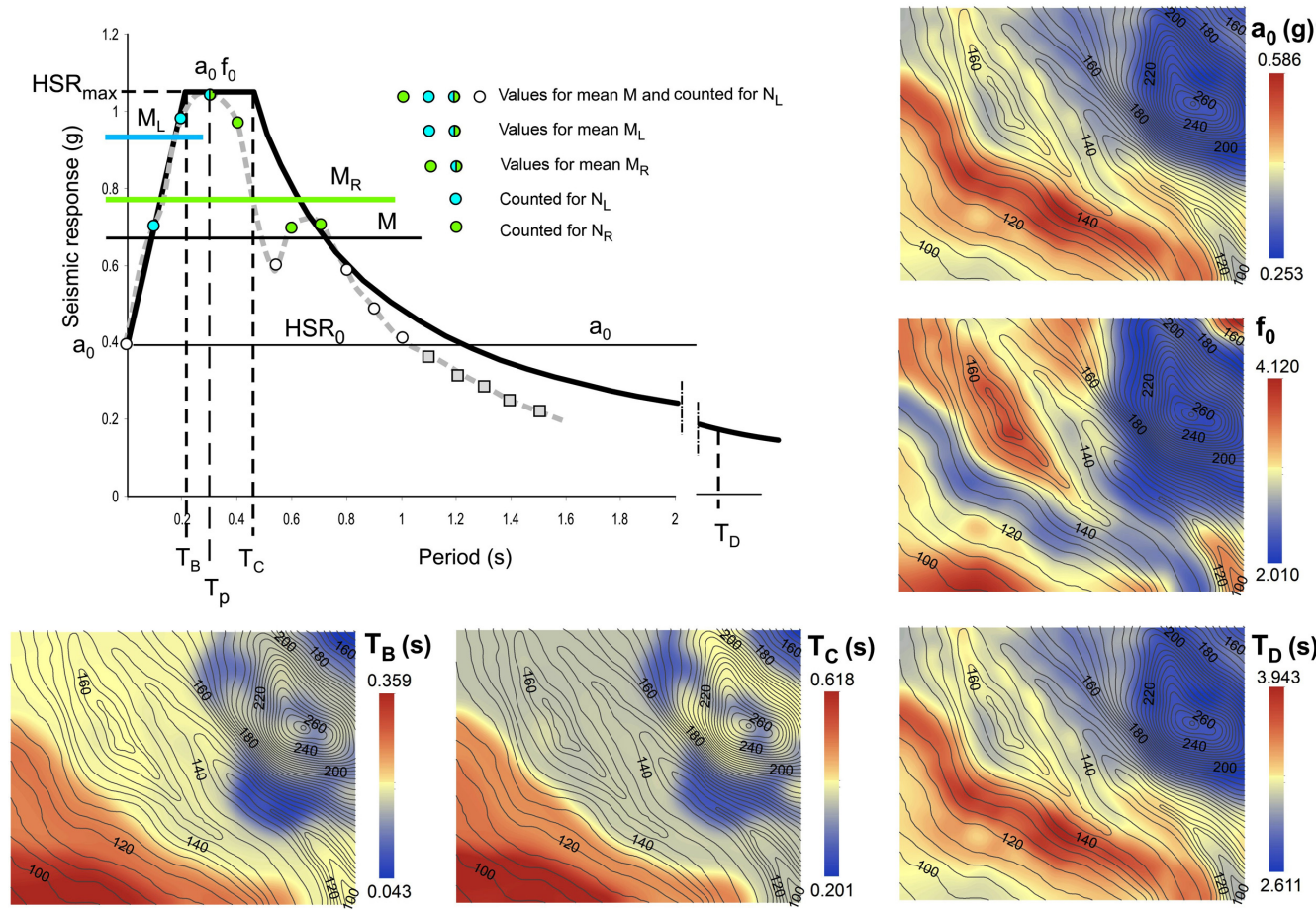

Figure 14. Enveloping model that creates the design spectrum; around it, the mapping distribution of the characteristic parameters of the design spectra, are shown.

\section{Discussion}

The SiSeRHMap methodology platform is composed of interdependent computational modules and sub-modules that in turn assume a crucial role in the prediction and therefore in the expected performance. Specifically, its seismic response map-sets are the result of a series of conventional/nonconventional procedures (hybrid) that use combined models that are simplified in different degrees in order to simulate the seismic response of more or less complex environments. On this subject and keeping in mind the theoretical bases as well as the validation cases, it seems appropriate to give here a complete overview of the strengths as well as the approximations and limitations of SiSeRHMap.

In general terms, the site seismic response of SiSeRHMap is defined as a 1-D stratigraphic effect, defined by trained meta-model, loaded with 3-D topographic effects in terms of the aggravation factor. An example is shown in Fig. 15; it regards the integration analysis of the Narni relief case considering the 1-D seismic response of a depth-decreased fracturing model computed with SiSeRHMap in a probolistic way assuming a single zone with a normal distribution (twenty combinations) of the shear wave velocity and thickness of layers. This data distribution is supported by the average uniform shear wave velocity proposed by Lovati et al. (2011) and Barani et. (2014) as well as by the local geological features (Storti and Salvini, 2001). The results shows a substantial matching with the experimental spectral ratio data referred to a strong motion data set (Sect. 4.1.1). However, in agreement with other authors (Sect. 1.2), the model may be limited when the mutual interaction of the two aforementioned effects appears considerable. For example, the possible influence of the topographic effect on the possible increasing of the non-linearity behaviour of the soils covering the reliefs is not contemplated, as well as the possible nonlinear response of the reliefs when these are constituted by soft materials.

Nevertheless, considering the aforementioned topics in reference to the single aspects of the SiSeRHMap model, it is possible to affirm that

- The GCM, which is the geometrical computation frame for the model, does not preset the geometrical limitation. It exploits the advantage of the multi-layer GISbuilding techniques. In the GCM, the lithodynamic unit is defined by non-linear/linear monotonic $V_{\mathrm{S}}$ depthdepending laws calibrated via a regression analysis of the selected and spatial diffused data. Taking into consideration this feature, the high standard deviations produced by localized clustered data may be diminished, inserting a new lithodynamic unit for this data.

- The multi-spectral maps regarding the stratigraphic seismic response are the result of meta-model processes on 1-D seismic responses regarding zonal 1-D trainer $V_{\mathrm{S}}-h$ profiles. This computational block is thus characterized by different critical nodes: (i) the seis- 


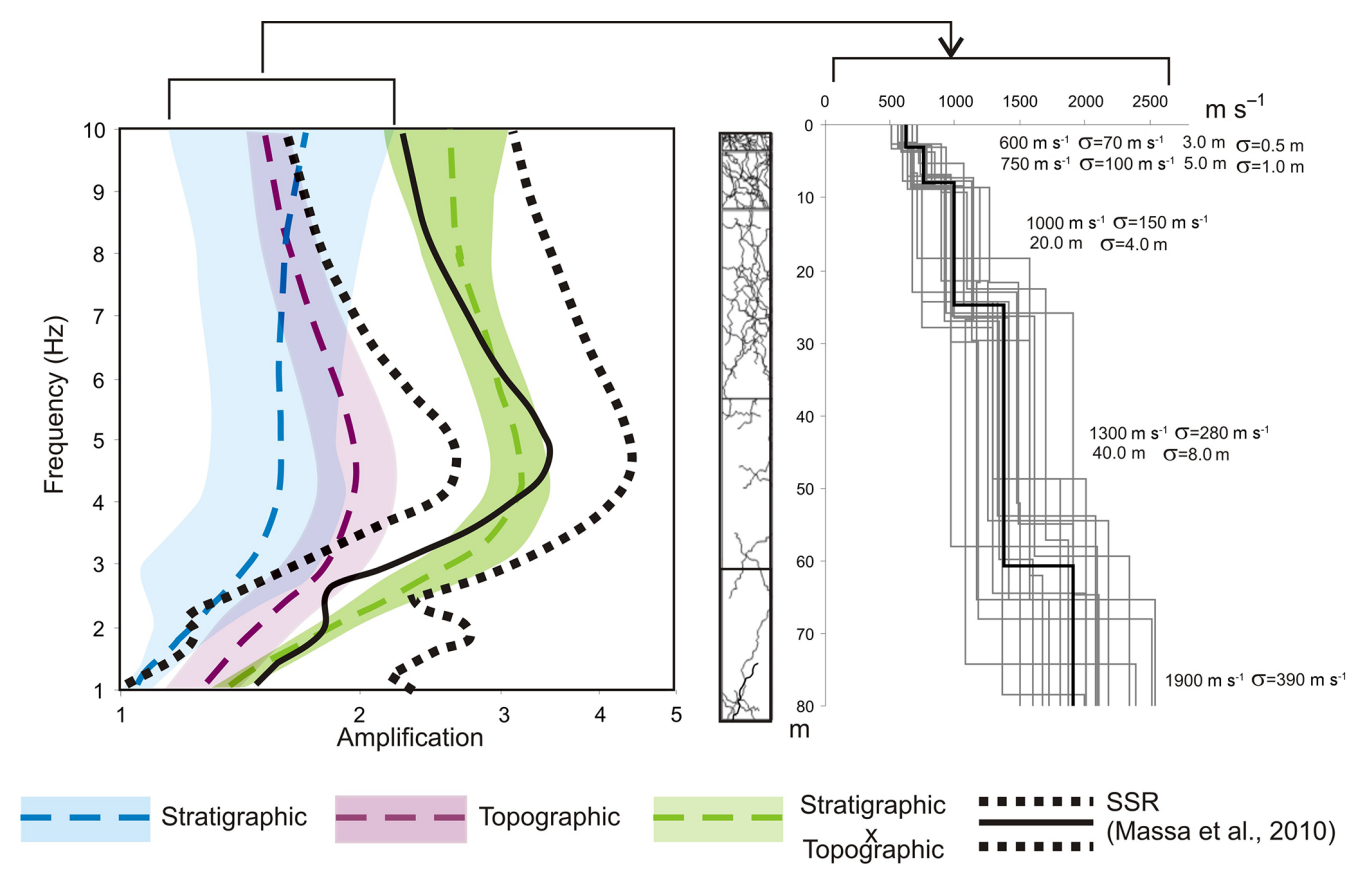

Figure 15. Seismic response by SiSeRHMap (linear analysis mode) in comparison to the SSR experimental analysis.

mic response is defined by a viscoelastic linear equivalent model with the same performance of similar models/codes (Fig. 5); the conservative aptitude degree of these models is the object of different case studies and suggestions (e.g. in Adampira et al., 2015; Zidan, 2015). (ii) The seismic responses obtained by the meta-model process are affected by checked trainer errors (intrinsic errors); in contrast the maps developed by the metamodel solving are affected by non-checked errors (prediction errors), that nevertheless have values comparable with the aforementioned checked errors. (iii) The maps generated by SiSeRHMap may suffer of substantial uncertainties when high complex subsoil features are present. The latter are summarized in the high slope degree of the interfaces $(L / H<8-10$ in Hasal and Iyisan, 2014) and in general by sharp variation of the buried morphology. On these effects, it is noted that 1$D$ seismic response seems to be underperformed mainly at the edge of the valley (Gelagoti et al., 2010). Future developments of SiSeRHMap will focused on this subject. (iv) Independence of site response to azimuth and the wave-incidence angles with subsoil interfaces.

- The frequency dependant topographic prediction model is based on the topographic response of simplified homogeneous regular reliefs. However, its reliability in the prediction for real cases has been ascertained (Fig. 13). Specifically, the prediction performances match the third party results deriving from different topographic frameworks and input motion sources, which are obtained both via numerical simulations and experimental analysis. The comparison, with the 3-D numerical simulation in homogeneous material, highlights (Fig. 13a) that SiSeRHMap's topographic spectral responses fall near the third quartile of normal output distributions for all different characterizing locations. The comparison with the results of the 3-D experimental analysis (Fig. 13b) confirm a relevant aptitude in the frequency range prediction of the topographic model. In addition, these cases highlight how epistemic uncertainty can be reduced assuming a calibrated $V_{S_{\text {Reg }}}$, which is obtained taking into consideration the experimental spectral ratio in the trial comparison analysis. For example, in the presence of a not well-known rigidity of the relief or in the presence of non-homogeneous material constituting the relief, a local frequency calibration, using also seismic signal noise or weak earthquake measurements (in single or multi-station recording mode), can be performed assuming a calibrate regional shear wave velocity that may be different from that used for depth rigid material (e.g. equivalent to $V_{S_{\text {Reg }}}$ ). To this regard, we can report that the computational times for the cases of Fig. 13a and b are approximately $24 \mathrm{~s}($ cell-size $=2 \mathrm{~m}$ ) and $3 \mathrm{~s}$ (cell-size $=5 \mathrm{~m})$, respectively.

However, some simplifications assumed here are common, in different degrees, to those used in simulation analysis performed by common physically based methods. Among these simplifications, there is the necessity to use simplified geometrical models, in addition to the necessity to parameterize these models by means of the interpretation and spatial distribution of the local data from field and/or laboratory sur- 
(a)
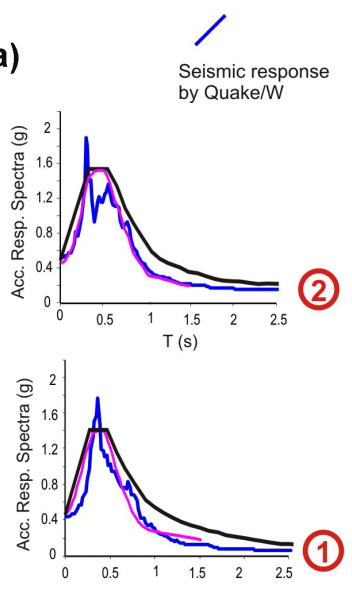

安
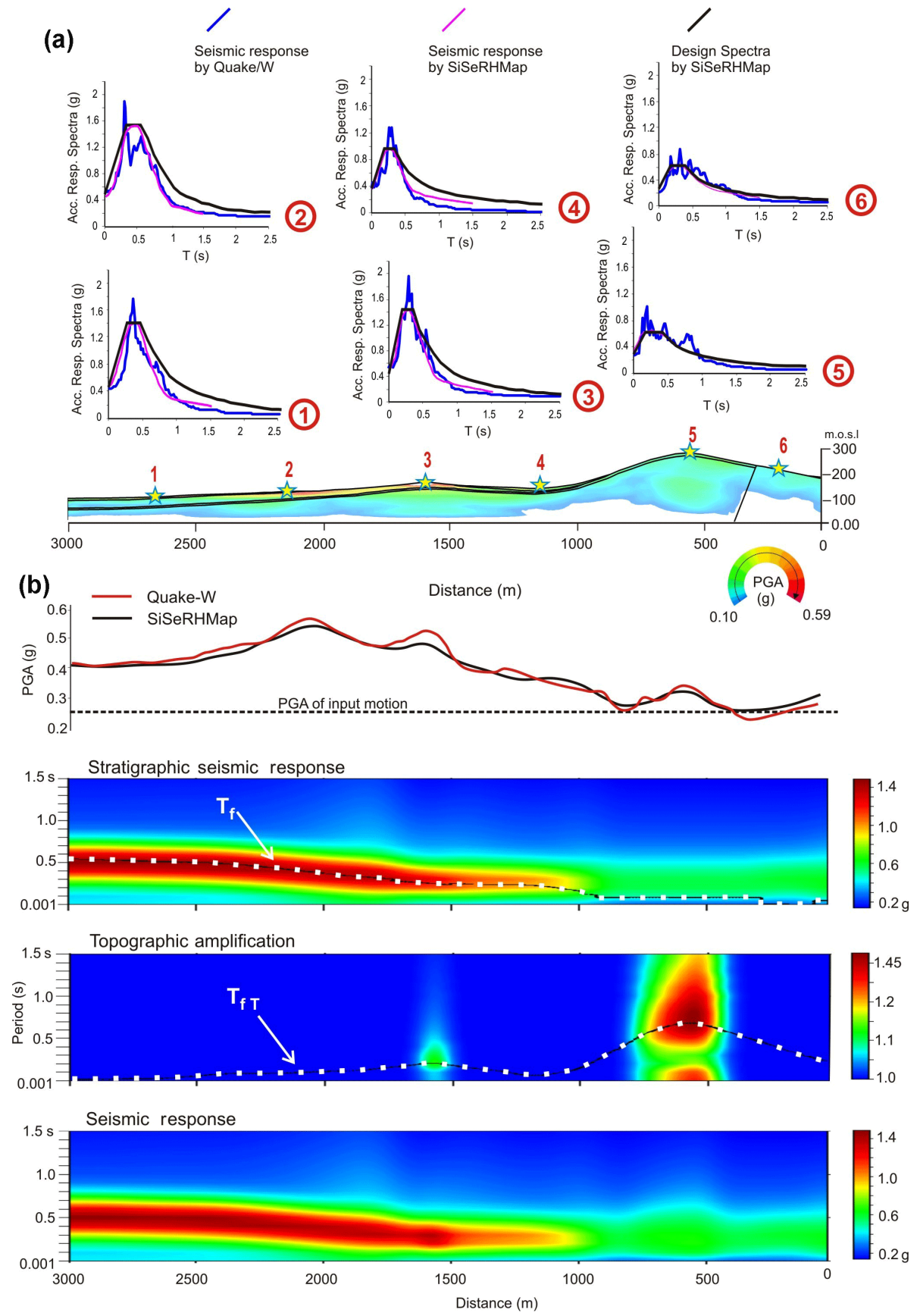

Figure 16. Comparison in some characterized points between the seismic response by SiSeRHMap and the Quake/W finite element method on a cross section showed in Fig. 1.

veys in order to define the lithodynamic model. In this context, SiSeRHMap is more efficacious in some large specific subjects that, in general, characterize the seismic response. This can be summarized in: (i) the use of the local adequate shear wave velocity of the lithodynamic units deriving from the statistical regression analysis; (ii) the development of georeferenced multi-spectral seismic response maps via the solving of meta-modelled smoothed responses that per- mit, in this way, a local (non generalized) computation of the design spectra expressed as parametric design spectral maps; (iii) the meta-model processes permit the obtainment of the trainer output data deriving from one or more input motions; (iv) the computation ability of every real 3-D topographic framework, which has proved to be more efficacious in comparison to 2-D numerical models in the analysis of 3-D relief shapes; (v) the possibility to include corrective 
practices guided by experimental analysis. For demonstration purposes, a final comparison between SiSeRHMap and a physically based numerical analysis code was performed on the synthetic recurrent scenario. The Quake/W (GeoStudio 2007) is a 2-D geotechnical finite element (FEM) software, which takes into consideration dynamic shear-straindependent viscoelastic material using dynamic linear equivalent analysis. This software offers the possibility to be parameterized using some of SiSeRHMap's input: the shear modules increase with effective vertical stress and consequently with depth; in addition it gives the possibility to assume the equivalent shear strain ratio in relation to magnitude. The comparison (Fig. 16) regards six points distributed along cross section A (trace in Fig. 1) in order to investigate different lithologies and topographic features. The input earthquake used in the comparison analysis is the same used in the stratigraphic response module (mod.3). This input motion is properly scaled in order to produce in the check point a spectrum coherent with the deconvoluted 1-D spectrum at the same depth. The check point is placed under the covered layer in the flat zone, while the mesh is assumed with different dimensions in relation to the thickness of the layers.

The comparison analysis highlights how the hybrid response is close in amplification as well as coherent in frequency to the response provided by exclusively physically based models solved by the 2-D FEM code. In this way, the aptitude of the hybrid model of SiSeRHMap seems to have a good compromise both for the definition of theoretical analytical response and for satisfying the exigency to provide the synthetic spectra shape required by building design.

\section{Conclusions}

SiSeRHMap introduces a new method, defined as "hybrid", which is capable of creating maps of seismic response based on concepts of simulation cases, training, and prediction.
The simulation (from mod.1 to mod.3) involves physicnumerical analysis consisting in a 1-D seismic response (mod.3), based on a linear-equivalent shear stress-strain model; this model performs on $V_{\mathrm{S}}-h$ profiles uniformly sampled in the GCM. The latter, in the first phase, is a structuredsynthetic representation of the subsoil by layered lithodynamic units (mod.1 and mod.2). The training is the core of the method due to the fact that it provides its hybrid evolution in the stratigraphic seismic response. In this way, the adaptive prediction model, Emul-spectra, seems to show robustness and efficacy features, while its accuracy is assured by the dedicated evolutionary algorithm (mod.4). The second phase of the GCM (mod.5) provides the mapped solution of the Emul-spectra model and the validated 3-D topographic prediction model, in order to produce map sets of hybrid seismic responses and their envelopment process with the design spectra. Therefore, the general model at the base of SiSeRHMap confers to it the attribute of a first computational program that associates consolidated techniques of stratigraphic seismic response with advanced techniques regarding numerical emulation models and their training. In this way, SiSeRHMap permits the obtainment of map data, which can be easily diffused and consulted.

\section{Code availability}

SiSeRHMap 1.0 is a free access code, it is available at http://www.geosmartapp.it where the trial version and full versions have been uploaded. The trial version is available and it only permits the running of the application case reported in the manuscript. The full version is freely available on demand inserting the password received at your mail after registration; this is an advanced version (ver. 1.1) with some suggestions proposed by referees. In the folder of the code, the user can also find the user guide and the input files that were used in the application case. 


\section{Appendix A: Stratigraphic Seismic Response module}

Module three computes the dynamic seismic response for a site model with infinitely extended horizontal covered layers assuming a vertical propagation of polarized shear waves stemming from a viscoelastic rigid bedrock (Fig. A1). The non-linear-viscoelastic strain that depends on the dynamic behaviour of soils constituting the covered layers is computed using the equivalent linear-viscoelastic analysis. Here, the base assumption is the 1-D linear-viscoelastic propagation of the shear wave in a homogeneous soil that is assumed as a Kelvin-Voigt solid in which the dynamic response is modelled using purely an elastic spring and a purely viscous dashpot (Kramer, 1996). For this model, the solution to the harmonic wave with a frequency $\omega$, that provides the displacement $u$, as a function of depth $z$, and time $t$ (Kramer, 1996), is

$u(z, t)=X \exp \left[j\left(\omega t+k^{*} z\right)\right]+Y \exp \left[j\left(\omega t-k^{*} z\right)\right]$,

where the first and second terms represent the incident and reflected wave travelling; therefore $X$ and $Y$ are, respectively, the amplitudes of the incident wave in the negative $z$-direction (upward) and reflected wave in the positive $z$ direction (downward). In addition, in Eq. (A1), $k^{*}$ is the complex wave number related to the complex shear modulus, $G^{*}$, damping ratio, $D$, and mass unit weight, $\rho$, of the soil, with

$k^{*}=\frac{\omega}{V_{\mathrm{S}}^{*}}=\frac{\omega}{\sqrt{\frac{G^{*}}{\rho}}}$,

taking into consideration that the critical damping ratio, $D$, is related to the viscosity, $\eta$, by

$\omega \eta=2 G D$.

Here, it is reasonable to assume that the dynamic parameters $G$ and $D$ are almost constant in the frequency range where the analysis is usually performed. Hence, it is possible to express the complex shear modulus in terms of the critical damping ratio instead of the viscosity:

$$
\begin{aligned}
G^{*}= & G+j \omega \eta=G\left(1-2 D^{2}+j 2 D \sqrt{1-D^{2}}\right) \\
& \cong G(1+2 j D),
\end{aligned}
$$

where $G$ can be taken as being independent from frequency.

Hence, from Eq. (A1), for the top and bottom interfaces of the $i$-layer with a thickness $h i$ (Fig. A1), it is possible to express the strain $\left[\left(u_{i}(0, t), u_{i}\left(h_{i}, t\right)\right]\right.$ in relation to the shear stress $\left[\left(\tau_{i}(0, t), \tau_{i}\left(h_{i}, t\right)\right]\right.$ in this way:

$$
\begin{aligned}
\tau_{i}(z, t)= & \left(G_{i}+j \omega \eta_{i}\right) \frac{\delta u_{i}}{\delta z}= \\
& j k_{i} G_{i}\left\{X \exp \left[j\left(\omega t+k_{i}^{*} z\right)\right]\right. \\
& \left.+Y \exp \left[j\left(\omega t-k_{i}^{*} z\right)\right]\right\} \exp (j \omega t) .
\end{aligned}
$$

Therefore, imposing the continuity condition in the interface, in generic time, $t$, the following occurs:

$u_{i}\left(h_{i}\right)=u_{i+1}(0)$ and $\tau_{i}\left(h_{i}\right)=\tau_{i+1}(0)$

obtaining the relations:

$$
\begin{aligned}
& X_{i} \exp \left(j k_{i}^{*} h_{i}\right)+Y_{i} \exp \left[-\left(j k_{i}^{*} h_{i}\right)\right]=X_{i+1}+Y_{i+1} \\
& k_{i}^{*} G_{i}^{*}\left(X_{i} \exp \left(j k_{i}^{*} h_{i}\right)+Y_{i} \exp \left[-\left(j k_{i}^{*} h_{i}\right)\right]\right) \\
& \quad=k_{i+1}^{*} G_{i+1}^{*}\left(X_{i+1}+Y_{i+1}\right) .
\end{aligned}
$$

For this later relation it is possible to express:

$\alpha_{i}=\frac{k_{i}^{*} G_{i}^{*}}{k_{i+1}^{*} G_{i+1}^{*}} \equiv \sqrt{\frac{\rho_{i} G_{i}^{*}}{\rho_{i+1} G_{i+1}^{*}}}$

and therefore to define the following recurrence formulation:

$$
\begin{aligned}
X_{i+1} & =\frac{1}{2}\left[X_{i}\left(1+\alpha_{i}\right) \exp \left(j k_{i}^{*} h_{i}\right)\right. \\
& \left.+Y_{i}\left(1-\alpha_{i}\right) \exp -\left(j k_{i}^{*} h_{i}\right)\right], \\
Y_{i+1} & =\frac{1}{2}\left[X_{i}\left(1-\alpha_{i}\right) \exp \left(j k_{i}^{*} h_{i}\right)\right. \\
& \left.+Y_{i}\left(1+\alpha_{i}\right) \exp -\left(j k_{i}^{*} h_{i}\right)\right] .
\end{aligned}
$$

At the top of the first layer in the free surface condition, the shear strength is $\tau_{1}(0)=0$. Hence, Eq. (A5) defines that the amplitude of incident $X_{1}$ and reflect $Y_{1}$ waves are equal. Therefore, once the shear module and damping in each layer is known, it is possible to compute the value of generic $X_{i}$ and $Y_{i}$ within the sequence for an assigned range of frequency. The computation is performed assuming the iterative recursive calculation starting from the free surface where $X_{1}=Y_{1}=1$ until the input (base) layer is reached. In this way, the transfer function for the incident and refract component of motion on the surface of the $i$ layer can be obtained from equations

$$
\begin{aligned}
& X_{i}=x_{i}(\omega) X_{1}, \\
& Y_{i}=y_{i}(\omega) Y_{1} \equiv y_{i}(\omega) X_{1} .
\end{aligned}
$$

Using Eq. (A1), the above transfer functions permit expressing the ratio of the amplitude of the harmonic motion in terms of displacement, velocity, and/or acceleration between two layers for each frequency assumed. Therefore, the resultant transfer function, $\operatorname{TF}(\omega)$ that defines the amplification between the rock surface associated with layer $(n)$ and the upper-surface of a cover layer $(i)$ or within the generic cover layer $(i)$, when a sub-layer division of the column is performed, is defined as

$\operatorname{TF}_{(n, i)}(\omega)=\frac{x_{i}(\omega)+y_{i}(\omega)}{x_{n}(\omega)+y_{n}(\omega)}$.

The above equation takes into consideration the amplification in relation to the input motion associated with an outcropping rock ( $n$-layer) where $X_{n}=Y_{n}$. In order to take into 


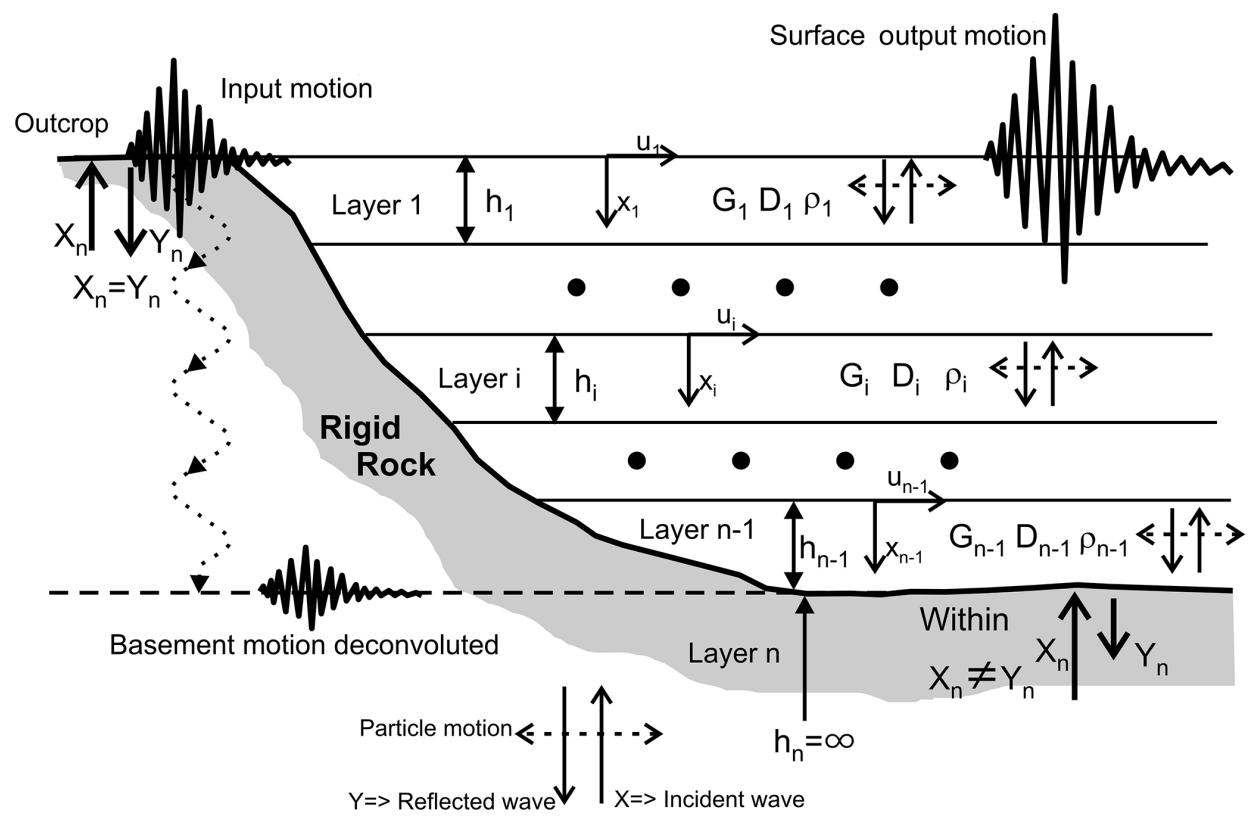

Figure A1. Stratigraphic amplification model (mod.3) consisting of a 1-D layered system composed of non-linear-viscoelastic soils covering the rigid viscoelastic bedrock.

account that the input motion is within a sequence at the base of the cover layer, a deconvolution operation must be performed. This operation assumes that the descending transfer function can be computed assuming that $X_{n} \neq Y_{n}$ at the base of the cover deposit. Hence, the transfer function between the upper surface of the layer or the sub-layer $(i)$ and bedrock surface $(n)$ is defined as

$$
\begin{aligned}
\operatorname{TF}_{(n, i)}(\omega)_{\text {input within }}= & \frac{x_{n}(\omega)+y_{n}(\omega)}{2 x_{n}(\omega)} \\
& \cdot \frac{x_{i}(\omega)+y_{i}(\omega)}{x_{n}(\omega)+y_{n}(\omega)} .
\end{aligned}
$$

In mod. 3 of SiSeRHMap, Eq. (A15) is set for the computation of $\operatorname{TF}_{(n, i)}(\omega)$ between the outcropping layer at the $z$-output surface and bedrock surface. In this way, the response at the $z$-output surface is computed by multiplying the Fourier amplitude spectrum of the input rock motion by the transfer function:

$\operatorname{OUTPUT}(\omega)=\operatorname{TF}_{(n, i)}(\omega) \cdot \operatorname{INPUT}(\omega)$

The Fourier amplitude spectra of the input motion is defined using the numpy.fft module in the scipy library that computes the 1-D $n$-point discrete Fourier transform (DFT) of a real-valued array by means of an efficient algorithm called the fast Fourier transform (FFT) (Cooley and Tukey, 1965; Press et al., 2007). In addition, this module computes the inverse of the $n$-point DFT for a real input matrix.

In relation to the strain dependent dynamic properties of the material, in the non-linear analysis, it is essential to know the strain values assumed during the motion. In the equivalent non-linear analysis, the dynamic module and damping is selected in the relative dynamic curve as a function of the strain level reached. This approach gives the possibility to use the transfer function for computing the shear strain, $\gamma$, which is calculated in the middle of layer; the shear strain transfer function amplifies the motion and converts acceleration into strain. In reference to the setting expressed by Eq. (A16), the shear strain transfer function is defined as

$$
\begin{aligned}
& \left(\operatorname{TF}_{(n, i)}(\omega)\right)_{\text {strain }}=\frac{\gamma(\omega, z)}{\ddot{u}_{n}(\omega)_{\text {outcropping }}} \\
& \quad=\frac{j k_{i}^{*}\left[X_{i} \exp \left(\frac{j k_{i}^{*} h_{i}}{2}\right)-Y_{i} \exp \left(-\frac{j k_{i}^{*} h_{i}}{2}\right)\right]}{-\omega^{2}\left(2 X_{n}\right)} .
\end{aligned}
$$

The strain Fourier amplitude spectrum is obtained applying the strain transfer function to the Fourier amplitude spectrum of the input motion. Consequently, from this spectrum, the time history strain is obtained using the Fourier time domain conversion. The level of the shear strain defined as equivalent to the dynamic effective strain is assigned in terms of ratio (equivalent shear ratio) in relation to the maximum shear strain.

The relationship between the equivalent strain obtained from Eq. (A17) and the dynamic shear strain dependent parameters assumed in the computation of Eq. (A15) entails that this latter is resolvable by exclusively using an iterative computation until the obtainment of a convergent solution starting from the assigned initial value of the damping ratio. Mod. 3 fits the data set regarding the shear modulus $G / G_{0}$, damping ratio $D(\%)$ and their relative strains, $\gamma$, using the following regression curves proposed by Yokota et 
al. (1981):

$\frac{G}{G_{0}}=\frac{1}{1+\alpha \gamma^{\beta}}$,

$D(\%)=D_{\max } \exp \left(-\lambda \frac{G}{G_{0}}\right)$.

Equations (A18) and (A19) are the non-linear logascending and log-descending curves, where $\alpha, \beta$, and after $D_{\max }$ are constant coefficients calibrated using the Levenberg-Marquardt algorithm in the computer aided version (Levenberg, 1944; Marquardt, 1963).

The seismic response spectra are defined by means of the widely used shock response spectra algorithm in which the seismic response spectrum is calculated using an acceleration time history as a common base input excitation to a serial array of single-degree-of-freedom (SDOF) systems. Each system is a damped harmonic oscillator characterized by mass, stiffness and damping. The damping of each system is commonly assumed. The natural frequency is an independent variable. Thus, the calculation is performed for an arbitrary number of independent SDOF systems, each with a unique natural frequency. The systems are considered to have no mass-loading effect on the base input excitation (Irvine, 2012, 2013).

The calculation method is carried out in the time domain via a convolution integral taking into consideration a base excitation with a ramp invariant function derivation of the digital recursive filtering relationship; the seismic response spectrum is the peak absolute acceleration response of each SDOF system to the time history base input (Smallwood, 1981). In the stratigraphic response module the acceleration response spectra function was developed starting from srs.py and using the tompy.py library module (Irvine, 2014).

\section{Appendix B: Evolutionary algorithm}

In the meta-model module (mod.4), the calibration of the Emul-spectra numerical model is performed by using the preprocessing evolutionary algorithm (EA) and subsequent optimization of data by means of the Levenberg-Marquardt algorithm (LMA) (Levenberg, 1944; Marquardt, 1963).

The LMA is implemented in Scipy Python's library as a "minpack" subroutine (http://www.math.utah.edu/software/ minpack/minpack/lmstr1.html). The LMA is a curve-fitting algorithm widely used to solve non-linear least-squares problems. However, as for many optimizer algorithms, the LMA finds local minima, which is not necessarily the global minima or optimal minima. This problem is due to some known aspects: (i) the large number of parameters; in fact a large number of parameters increases the search-hyperspace dimensions and therefore a higher number of local minimum values are developed; (ii) the parameters differ from each other by some orders of magnitudes; (iii) the slowed convergence when the least-squared function is very flat and the global minimum is located in the "narrow canyon". Therefore, the non-uniqueness of an inverse solution and slowness in convergence are very sensitive to initial guesses.

The EA (Fig. B1) is an evolutionary computational metaheuristic method that consists in two breeding levels in which the first level generates, starting from initial guesses parameters (grandparents values) the offsprings (parents solutions) which are naturally selected for breeding (evolution) in the second level. Consequently, in this level, the next generations are reproductions in a new generation (fourth in $\mathrm{SiS}$ eRHMap) from better parents; these offsprings are no longer subjected to natural selection but a new form of elitism is carried out. Using the root mean squared error in the definition of fitness, the reaching of convergence between the fitting minimum error, $E_{\min }$, and the increasing error target $E_{\text {targ }}$, determines the satisfaction of the algorithm termination criterion and an optimized minima error solution should be reached after having tried to escape the unsatisfactory local minima error solutions. The numerical parameters obtained in this way are the best initial guesses in the LMA optimize process.

In the first breeding level, the parent solutions $\left(x_{1, i}, \ldots, x_{8, i}\right)$ are generated in a normal distribution from given mean values $\left(x_{1}, \ldots, x_{8}\right)$, defined as grandparents, and standard deviation $\left(\delta_{1}, \ldots, \delta_{8}\right)$. The grandparents values differ by up to 3-4 orders of magnitude and are the results of the sensitive analysis performed on many meta-model cases; these values are reported as default but they can be changed.

When the $i$ th parent population is generated, its performance in fitness, $E_{i}$, is compared with the best performance of the previous parent populations defined by the minimum current error $E_{\min }$, and with the current error target $E_{\mathrm{targ}}$. If $E_{i}$ is equal or less than $E_{\mathrm{targ}}$, the problem is already solved in the first breeding level. This occurs when there is a premature convergence (Eq. 15), due to the assuming of a high value of the starting $E_{\mathrm{targ}}$, or when indeed a good solution is found (rarely). However, if $E_{i}$ is greater than $E_{\min }$, the iterating process continues and a new parent population is generated; in contrast, if $E_{i}$ is less than $E_{\min }$, the parent population passes to the second breeding level and the $E_{\min }$ assumes the current $E_{i}$ value. The current $E_{\text {min }}$ values are kept until the assigned iteration value, $B$, is reached.

In the second breeding level, the $k$ th descending populations can be generated; starting from $k=0, j$-iterate solutions are procreated in normal distribution series assuming as mean values $\left(x_{1, j, 0}, \ldots, x_{8, j, 0}\right)$, that are the elect parent population $\left(x_{1, i}, \ldots x_{8, i}\right)$ deriving from the first level, and standard deviation $\left(\delta_{1}, \ldots, \delta_{8}\right)$. The procreation of new $j$-populations continues until a new and better error is found or until an assigned $j$-iteration value, $C$, is reached. In the first case, the population is a new generation and it assumes the role of $k$ th procreator having mean values, $x_{1, j, k}, \ldots, x_{8, j, k}$, and a standard deviation $\delta_{1} / k, \ldots, \delta_{8} / k$. The $k$-iteration of the aforementioned loop continues up until an assigned number of 


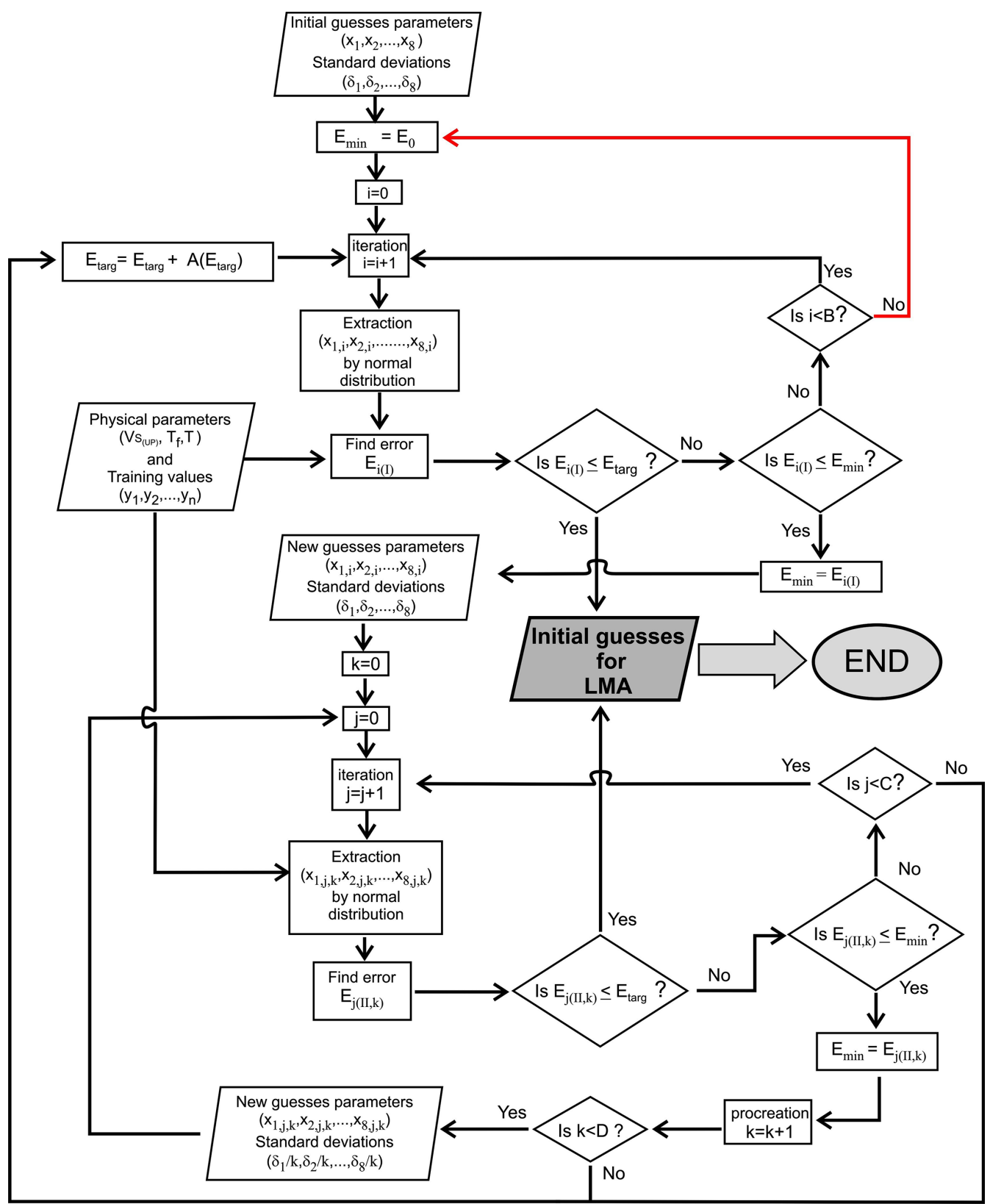

Figure B1. The evolutionary algorithm (EA) scheme: $x$ and $\delta$ are the mean and the standard deviation in normal distribution; I and II indicate the first and the second phase; $i, j$ are the generic populations; $k$ is the ranking of the generation in the second phase; $E_{0}$ is the initial error (100); $E_{\min }$ is the current error; $E_{\mathrm{targ}}$ is the initial error target, it depends on the number of lithodynamic units in the $V_{\mathrm{S}}-h$ trainer model and the number of trainer models ( 0.005 to 0.05$) ; A$ is the increased ratio of the $E_{\operatorname{targ}}(0.02) ; B$ is the number of the generated population (2000) before the mass extinction (red flow line); $C$ is the max number of populations permitted in a generation of the second level (100); $D$ is the number of the generation in the second phase (4).

generations, $D$, is reached; if the convergence is not found in this process, in addition to the reaching of $C$, the process returns to the first level and the error target is increased by an A value. When the process returns to the first level, the minimum error assumes the value of the last minimum error found in this level. However, the minimum error and target error are reset when $B$ in the $i$-iteration value is reached.
The optimal solution does not contemplate absolute minimums, being that for one or more elements (inter-space vectors), the solution tends to be infinite. For this reason, a solution that gives values that do not exceed a greatness of $10^{5}$, is considered optimal. 


\section{Appendix C: Topographic amplification}

SiSeRHMap permits a definition of the basal surface of relief (BSR) in relation to the general setting of the topographic area. The BSR is a flat or not flat surface that tries to isolate local idealized relief conditions, and its greater efficacy occurs when one ridge is seen as such in the 2-D relief scanning in at least one of the directions. Furthermore, the area assumed in the topographic amplification analysis should match the aforementioned requirement. Hence, a dedicated algorithm defines

a. the BSR as a wary surface. The algorithm performs the numerical scanning in $X$ and $Y$ (east-west and northsouth) directions choosing the maximum and minimum elevation value $\mathrm{Ex}_{\max }, \mathrm{Ey}_{\max }$ and $\mathrm{Ex}_{\min }, \mathrm{Ey}_{\min }$. Therefore, taking into consideration the generic map position $(x, y) \in(X, Y)$ the height of the relief is defined as

a1. $H=\min \left[\left(E x, y-E x_{\min }\right),\left(E x, y-E y_{\min }\right)\right]$ $H_{\max }=\min \left[\left(E x_{\max }-E x_{\min }\right),\left(E y_{\max }-E y_{\min }\right)\right]$

a2. $H=\max \left[\left(E x, y-E x_{\min }\right),\left(E x, y-E y_{\min }\right)\right]$ $H \max =\min \left[\left(E x_{\max }-E x_{\min }\right),\left(E y_{\max }-E y_{\min }\right)\right]$ b. the BRS as a plain surface with elevation, $E_{\text {flat }}$, results from an average elevation of the flat zones. These latter are so defined when they show a slope $i<5^{\circ}$ and curvature $-0.05 \leq c \leq 0.05$.

b1. $H=E x, y-E_{\text {flat }}$ $H \max =\max \left[\left(E_{\max }-E_{\text {flat }}\right),\left(E y_{\max }-E_{\text {flat }}\right)\right]$ 


\section{The Supplement related to this article is available online at doi:10.5194/gmd-9-1567-2016-supplement.}

Author contributions. The SiSeRHMap methodology was created by G. Grelle with the collaboration of L. Bonito. G. Grelle was also the developer and the project creator of the source code. L. Bonito provided support in the geographic information system and in the development of the GIS cubic model. A. Lampasi supported the planning of the evolutionary algorithm. P. Revellino and L. Guerriero collaborated in the definition of the synthetic recurrent scenario (SRS) and in the textual user interface and manuscript editing. G. Sappa and F. M. Guadagno were research and project coordinators.

Acknowledgements. We are a really grateful to the topical editor (Lutz Gross) and the anonymous reviewers for their constructive comments and suggestions, which helped us to improve the manuscript and show the potential of the model.

Edited by: L. Gross

\section{References}

Adampira, M., Alielahi, H., Panji, M., and Koohsari, H.: Comparison of equivalent linear and nonlinear methods in seismic analysis of liquefiable site response due to near-fault incident waves: a case study, Arabian Journal of Geosciences, 8, 3103-3118, 2015.

Ashford, S. A., Sitar, N., Lysmer, J., and Deng, N.: Topographic effects on the seismic response of steep slopes, B. Seismol. Soc. Am., 87, 701-709, 1997.

Assimaki, D., Kausel, E., and Gazetas., G.: Soil-dependent topographic effects: a case study from the 1999 Athens earthquake, Earthquake Spectra, 21, 929-966, 2005.

Athanasopoulos, G. A., Pelekis, P. C., and Leonidou, E. A.: Effects of surface topography on seismic ground response in the Egion (Greece) 15 June 1995 earthquake, Soil Dyn. Earthq. Eng., 18, 135-149, 1999.

Barani, S., Massa, M., Lovati, S., and Spallarossa, D.: Effects of surface topography on ground shaking prediction: Implications for seismic hazard analysis and recommendations for seismic design, Geophys. J. Int., 197, 1551-1565, 2014.

Bardet, J. P., Ichii, K., and Lin, C. H.: EERA: A computer program for Equivalent-linear Earthquake site Response Analyses of layered soil deposits, Department of Civil Engineering, University of Southern California, Los Angeles, California, 2000.

Bianchi Fasani, G., Cavinato, G. P., Petitta M., Scarascia Mugnozza, G., and Voltaggio, M.: The geological model of Celano town area for seismic microzonation activities, Soil Dyn. Earthq. Eng., 28, 978-985, 2008.

Borcherdt, R. D.: Estimates of Site - Dependent Response Spectra for Design (Methodology and Justification), Earthq. Spectra, 10, 617-653, 1994.
Bostenaru Dan, M.: Multidisciplinary co-operation in building design according to urbanistic zoning and seismic microzonation, Nat. Hazards Earth Syst. Sci., 5, 397-411, doi:10.5194/nhess-5397-2005, 2005.

Bouchon, M., Schultz, C. A., and Toksoz, M. N.: Effect of threedimensional topography on seismic motion, J. Geophys. Res., 101, 5835-5846, 1996

Cardarelli, E., Ceracato, M., de Nardis, R., Di Filippo, G., and Milana, G.: Geophysical investigations for seismic zonation in municipal areas with complex geology: The case study of Celano, Italy, Soil Dyn. Earthq. Eng., 28, 950-963, 2008.

Çelebi, M.: Topographical and geological amplifications determined from strong-motion and aftershocks records of the 3 march 1985 Chile earthquake, B. Seismol. Soc. Am., 77, 11471167, 1987.

Compagnoni, M., Pergalani, F., and Boncio, P.: Microzonation study in the Paganica-San Gregorio area affected by the April 6, 2009 L'Aquila earthquake (central Italy) and implications for the reconstruction, B. Earthq. Eng., 9, 181-198, 2011.

Cooley, J. W. and Tukey, J. W.: An algorithm for the machine calculation of complex Fourier series, Math. Comput., 19, 297-301, 1965.

Del Gaudio, V. and Wasowski, J.: Directivity of slope dynamic response to seismic shaking, Geophys. Res. Lett., 34, L12301, doi:10.1029/2007GL029842, 2007.

Del Prete, M., Guadagno, F. M., and Scarascia-Mugnozza, G.: Earthquake induced damage in an historic area: the SeptemberOctober 1997 seismic sequence which affected Assisi, Central Italy, B. Eng. Geol. Environ., 57, 101-109, 1998.

Durand, S., Gaffet, S., and Virieux, J.: Seismic diffracted waves from topography using 3-D discrete wavenumberboundary integral equation simulation, Geophysics, 64, 572578, doi:10.1190/1.1444564, 1999.

Fan, F. M., Fleischmann, A. S., Collischonn, W., Ames, D. P., and Rigo, D.: Large-scale analytical water quality model coupled with GIS for simulation of point sourced pollutant discharges, Environ. Modell. Softw., 64, 58-71, 2015.

FEMA 356: Prestandard and Commentary for the Seismic Rehabilitation of Buildings, prepared by theBuilding Seismic Safety Council for the Federal Emergency Management Agency, 2000, Washington, DC, 2000.

Gallipoli, M. R., Bianca, M., Mucciarelli, M., Parolai, S., and Picozzi, M.: Topographic versus stratigraphic amplification: Mismatch between code provisions and observations during the L'Aquila (Italy, 2009) sequence, B. Earthq. Eng., 11, 1325-1336, 2013.

Ganapathy, G. P.: First level seismic microzonation map of Chennai city - a GIS approach, Nat. Hazards Earth Syst. Sci., 11, 549559, doi:10.5194/nhess-11-549-2011, 2011.

Gelagoti F., Kourkoulis R., Anastasopoulos I., Tazoh T., and Gazetas G.: Seismic wave propagation in a very soft alluvial valley: sensitivity to ground-motion details and soil nonlinearity and generation of a parasitic vertical component, B. Seismol. Soc. Am., 100, 3035-3054, 2010.

Geli, L., Bard, P. Y., and Jullen, B.: The effect of topography on earthquake ground motion: a review and new results, B. Seismol. Soc. Am., 78, 42-63, 1988. 
Grasso, S. and Maugeri, M.: The Seismic Microzonation of the City of Catania (Italy) for the Etna Scenario Earthquake $(M=6.2)$ of 20 February 1818, Earthq. Spectra, 28, 573-594, 2012.

Grelle, G. and Guadagno, F. M.: Regression analysis for seismic slope instability based on Double Phase Viscoplastic sliding model of the rigid block, Landslides, 5, 583-597, 2013.

Grelle, G., Revellino, P., and Guadagno, F. M.: Methodology for seismic and post-seismic stability assessing of natural clay slope based on a visco-plastic behavioural model in simplified dynamic analysis, Soil Dyn. Earthq. Eng., 12, 1248-1260, 2011.

Grelle, G., Bonito, L., Revellino, P., Guerriero, L., and Guadagno, F. M.: A hybrid model for mapping simplified seismic response via a GIS-metamodel approach, Nat. Hazards Earth Syst. Sci., 14, 1703-1718, doi:10.5194/nhess-14-1703-2014, 2014.

Gutierrez, C. and Singh, S. K.: A site effect study in Acapulco, Guerrero, Mexico: comparison of results from strong-motion and microtremor data, Seismological Society of America, 82, 642659, 1992.

Hasal, M. E. and Iyisan, R.: A numerical study on comparison 1D and 2D seismic responses of a basin in Turkey, America Journal of Civil Engineering, 2, 123-133, 2014.

Hashemi, M. and Alesheikh, A. A.: Development and implementation of a GIS-based tool for spatial modeling of seismic vulnerability of Tehran, Nat. Hazards Earth Syst. Sci., 12, 3659-3670, doi:10.5194/nhess-12-3659-2012, 2012.

Hassanzadeh, R., Nedović-Budić, Z., Alavi Razavi, A., Norouzzadeh, M., and Hodhodkian, H.: Interactive approach for GISbased earthquake scenario development and resource estimation (Karmania hazard model), Comput. Geosci., 51, 324-338, 2013.

Hong, S., Yu, X., Park, S. K., Choi, Y.-S., and Myoung, B.: Assessing optimal set of implemented physical parameterization schemes in a multi-physics land surface model using genetic algorithm, Geosci. Model Dev., 7, 2517-2529, doi:10.5194/gmd7-2517-2014, 2014.

Hough, S. E., Altidor, J. R., Anglade, D., Given, D., Janvier, M. G., Maharrey, J. Z., Meremonte, M., Mildor, B. S.-L., Prepetit, C., and Yong, A.: Localized damage caused by topographic amplification during the 2010 M7.0 Haiti earthquake, Nat. Geosci., 3, 778-782, 2010.

Idriss, I. M. and Sun, J. I.: User's Manual for SHAKE91, Center for Geotechnical Modeling, Department of Civil Engineering, University of California, Davis, 1992.

Imai, T. and Tonouchi, K.: Correlation of N-value with S-wave velocity and shear modulus, in: Proceedings of the 2nd European Symposium of Penetration Testing, Amsterdam, 57-72, 1982.

Irvine T.: An introduction to the shock response spectrum - Revision S, available at: http://www.vibrationdata.com/tutorials2/srs_ intr.pdf (last access: 21 April 2016), 2012.

Irvine, T.: Derivation of the Filter Coefficients for the Ramp Invariant Method as Applied to Base Excitation of a Single-degree-of-Freedom System - Revision B, available at: http://citeseerx.ist.psu.edu/viewdoc/download;jsessionid= 938D2487CE3E688D1E99D724A02A1257?doi=10.1.1.353. 8129\&rep=rep1\&type=pdf (last access: 21 April 2016), 2013.

Irvine T.: Vibration data Pyrotechnic Shock and Shock Response Spectrum Page, available at: http://www.vibrationdata.com/SRS. htm (last access: 21 April 2016), 2014.
Jimenez, M. J., Garcia-Fernadez, M., Zonno, G., and Cella, F.: Mapping Sol Effects In Barcelona, Spain, Through An Integrated Gis Environment, Soil Dyn. Earthq. Eng., 19, 289-301, 2000.

Kawase, H. and Aki, K.: Topography effect at the critical SV-wave incidence: possible explanation of damage pattern by the Whittier Narrows, California, earthquake of 1 October 1987, B. Seismol. Soc. Am., 80, 1-22, 1990.

Keçeli, A.: Soil parameters which can be determined with seismic velocities, Jeofizik, 16, 17-29, 2012.

Kienzle, A., Hannich, D., Wirth, W., Ehret, D. Rohn, J., Ciugudean, V., and Czurda K.: A GIS-based study of earthquake hazard as a tool for the microzonation of Bucharest, Eng. Geol., 87, 13-32, 2006.

Kokošin, J. and Gosar, A.: Seismic Microzonation of Breginjski Kot (NW Slovenia) Based on Detailed Engineering Geological Mapping. Hindawi Publishing Corporation, The Scientific World Journal, 2013, 12 pp., 2013.

Kolat, Ç., Doyuran, V., Ayday, C., and Lütfi Süzen M.: Preparation of a geotechnical microzonation model using Geographical Information Systems based on Multicriteria Decision Analysis, Eng. Geol., 87, 241-255, 2006

Kottke, A. and Rathje, E. M.: "Strata", available at: https://nees.org/ resources/strata (last access: 21 April 2016), 2010.

Kottke, A. R. and Rathje, E. M.: "Technical manual for Strata". Report 2008/10, Pacific Earthquake Engineering Research [PEER] Center, Berkeley, California, 95 pp., 2008.

Kramer, S. L.: Geotechnical Earthquake Engineering, Prentice Hall, 653 pp., 1996.

Lachet, C., Hatzfeld, D., Bard, P. Y., Theodulidis, N., Papaioannou, C., and Savvaidis, A.: Site effects and microzonation in the City of Thessaloniki (Greece) comparison of different approaches, B. Seismol. Soc. Am., 86, 1692-1703, 1996.

Lampasi, D. A., Podestà, L., and Carbone, P.: Synthesis of Binary Test Sequences with Good Spectral Properties Using an Evolutionary Algorithm, Elsevier Measurement, 39, 245-251, 2006.

Levenberg, K.: A Method for the Solution of Certain Problems in Least Squares, Q. Appl. Math., 2, 164-168, 1944.

Lovati, S., Bakavoli, M. K. H., Massa, M., Ferretti, G., Pacor, F., Paolucci, R., Haghshenas, E., and Kamalian, M.: Estimation of topographical effects at Narni ridge (Central Italy): Comparisons between experimental results and numerical modelling, B. Earthq. Eng., 9, 1987-2005, 2011.

Lum, K. and Yan, L.: In situ measurements of dynamic soil properties and liquefaction resistances of gravelly soils at Keenleyside Dam. Proc., Ground Failures under Seismic Conditions, ASCE Geotechnical Special Publication, Atlanta, No. 44, 221240, 1994.

Marquardt, D.: An Algorithm for Least-Squares Estimation of Nonlinear Parameters, SIAM J. Appl. Math., 11, 431-441, 1963.

Massa, M., Lovati, S., D’Alema, E., Ferretti, G., and Bakavoli, M.: An experimental approach for estimating seismic amplification effects at the top of a ridge, and the implication for groundmotion predictions: The case of Narni, Central Italy, B. Seismol. Soc. Am., 100, 3020-3034, 2010.

Maufroy, E., Cruz Atienza, V. M., and Gaffet, S.: A Robust Method for Assessing 3-D Topographic Site Effects: A Case Study at the LSBB Underground Laboratory, France, Earthq. Spectra, 28, 1097-1115, 2012. 
Maufroy, E., Cruz Atienza, V. M., Cotton, F., and Gaffet, S.: Frequency Scaled Curvature as a Proxy for Topographic Site Effect Amplification and Ground Motion Variability, B. Seismol. Soc. Am., 105, 354-367, 2015.

Milana, G., Azzara, R. M., Bertrand, E., Bordoni, P., Cara, F., Cogliano, R., Cultrera, G., Di Giulio, G., Duval, A. M., Fodarella, A., Marcucci, S., Pucillo, S., Régnier, J., and Riccio, G.: The contribution of seismic data in microzonation studies for downtown L'Aquila, B. Earthqu. Eng., 9, 741-759, 2011.

Moscatelli, M., Pagliaroli, A., Cavinato, G. P., Castenetto, S., and Naso, G.: Seismic microzonation of Palatine hill, Roman Forum and Coliseum Archaeological Area, B. Earthq. Eng., 12, 12691275, 2014.

Nath, S. K.: Seismic Hazard Mapping and Microzonation in the Sikkim Himalaya through GIS Integration of Site Effects and Strong Ground Motion Attributes, Nat. Hazards, 31, 319-342, 2004.

Nguyen H. T., Fleurisson, J. A., and Cojean, R.: Evaluation of topography site effect in slope stability under dynamic loading, Vienna Congress on Recent Advances in Earthquake Engineering and Structural Dynamics, Vienna, Austria, 10 pp., 2013.

Ohta, Y. and Goto, N.: Empirical shear wave velocity equations in terms of characteristic soil indexes, Earthq. Eng. Struct. D., 6, 167-187, 1978.

Ordónez, G. A.: SHAKE 2000: A computer program for the 1$\mathrm{D}$ analysis of thegeotechnical earthquake engineering problem, available at: http://www.geomotions.com/modules.php?name= Content\&pa=showpage \&pid=8 (last access: 21 April 2016), 2009.

Paolucci, R.: Amplification of earthquake ground motion by steep topographic irregularities, Earthq. Eng. Struct. D., 31, 18311853, 2002.

Papadimitriou, A. G., Antoniou, A. A., Bouckovalas, G. D., and Marinos, P. G.: Methodology for automated GIS-aided seismic microzonation studies, Comput. Geotech., 35, 505-523, 2008.

Pischiutta, M., Cultrera, G., Caserta, A., Luzi, L., and Rovelli, A.: Topographic effects on the hill of Nocera Umbra, central Italy, Geophys. J. Int., 182, 977-987, 2010.

Press, W., Teukolsky, S., Vetterline, W. T., and Flannery, B. P.: Numerical Recipes: The Art of Scientific Computing, chapter 1213, Cambridge Univ. Press, Cambridge, UK, 2007.

Reed, S. E., Boggs, J. L., and Mann, J. P.: A GIS tool for modeling anthropogenic noise propagation in natural ecosystems, Environ. Modell. Softw., 37, 1-5, 2012.

Rodríguez-Marek, A., Bray, J. D., and Abrahamson, N. A.: An empirical geotechnical seismic site response procedure, Earthq. Spectra, 17, 65-87, 2001.

Rollins, K. M., Evans, M. D., Diehl, N. B., and Daily, W. D.: Shear modulus and damping relationships for gravels, J. Geotech. Geoenviron., 124, 396-405, 1998.

Sánchez-Sesma, F. J.: Diffraction of elastic waves by threedimensional surface irregularities, B. Seismol. Soc. Am., 73, 1621-1636, 1983.
Schnabel, P. B., Lysmer, J., and Seed, H. B.: SHAKE: A Computer Program for Earthquake Response Analysis of Horizontally Layered Sites, Report No. EERC 72-12, College of Engineering, University of California, Berkeley, CA, 88 p., 1972.

Smallwood, D. O.: An Improved Recursive Formula for Calculating Shock Response Spectra, Shock and Vibration Bulletin, 51, 1981.

Soares, J., Kousa, A., Kukkonen, J., Matilainen, L., Kangas, L., Kauhaniemi, M., Riikonen, K., Jalkanen, J.-P., Rasila, T., Hänninen, O., Koskentalo, T., Aarnio, M., Hendriks, C., and Karppinen, A.: Refinement of a model for evaluating the population exposure in an urban area, Geosci. Model Dev., 7, 1855-1872, doi:10.5194/gmd-7-1855-2014, 2014.

Sokolov, V. Y. and Chernov, Y. K.: Probabilistic Microzonation of Urban Territories: A Case of Tashkent City, Pure Appl. Geophys., 158, 2295-2311, 2011.

Storti, F. and Salvini, F.: The evolution of a model trap in the central Apennines, Italy: Fracture patterns, fault reactivation and development of cataclastic rocks in carbonates at the Narni Anticline, J. Petrol. Geol., 24, 171-190, 2001.

Spudich, P., Hellweg, M., and Lee, W. H. K.: Directional topographic site response at Tarzana observed in aftershocks of the 1994 Northridge, California, earthquake: Implications for mainshock motions, B. Seismol. Soc. Am., 86, 193-208, 1996.

Todd, D. R. and Harris, J. R.: De Facto Microzonation Through the Use of Soils Factors in Design Triggers, International Conference on Seismic Zonation, 5th. Proceedings, 17-19 October 1995, Nice, France, Quest Editions, France, 510-517, 1995.

Turk, T., Gümüşay, U., and Tatar, O.: Creating infrastructure for seismic microzonation by Geographical Information Systems (GIS): A case study in the North Anatolian Fault Zone (NAFZ), Comput. Geosci., 43, 167-176, 2012.

Wang, C., Duan, Q., Gong, W., Ye, A., Di, Z., and Miao, C.: An evaluation of adaptive surrogate modeling based optimization with two benchmark problems, Environ. Modell. Softw., 60, 167-179, 2014.

Yazdi, J. and Neyshabouri S.: Adaptive surrogate modeling for optimization of flood control detention dams, Environ. Modell. Softw., 61, 106-120, 2014.

Yokota, K., Imai, T., and Kanemori, T.: Dynamic deformation characteristics of soils determined by laboratory tests, OYO Technical Report, 3, 13-36, 1981.

Zidan, A. F.: Modelling of soil damping for seismic ground response by nonlinear finite element analysis, Proc. of the Third Intl. Conf. on Advances in Civil and Structural Engineering, 2015. 